\title{
LES MEULES DU MOULIN À MINERAI MÉDIÉVAL DE BRANDES-EN-OISANS
}

\author{
N. MiNVIELLE LAROUSSE
}

\begin{abstract}
Mots-clés Moyen Âge, Moulin, meule à minerai, mines, minéralurgie, Brandes, argent. Keywords Middle Ages, mill, ore millstone, mines, mineral processing, Brandes, silver.

Résumé Un corpus de 125 meules et fragments de meules associé à 309 éclats a été mis au jour en 2013 et 2014 lors de la fouille d'un moulin à minerai hydraulique sur le site de Brandes-en-Oisans (France, Isère, Huez). Intégré dans la chaîne opératoire des métaux polymétalliques (plomb, cuivre, argent), l'étape du broyage se place entre le concassage et le lavage. La majeure partie des meules est datée de la première moitié du XIII siècle; seules quelques-une en remploi dans le bâti sont légèrement antérieures à 1210. L'étude typologique met en évidence des outils homogènes d'un diamètre de 80 à $130 \mathrm{~cm}$. Elles se caractérisent par des rayons et des stries, tout en étant pour la plupart dissymétriques, usées et fragmentées. Exemple d'un usage industriel de l'énergie hydraulique, ces meules s'intègrent dans les premières études typologiques des outils de mouture médiévaux.
\end{abstract}

\begin{abstract}
125 whole and fragmented millstones associated with 309 small millstone flakes (chipped off during use) were unearthed in the 2013 and 2014 excavations of a hydraulic ore mill at Brandes-en-Oisans (Huez, Isère, France). Ore grinding, a step taking place between crushing and washing, is part of the operational sequence of polymetallic metals (lead, copper, silver) production. Most of the millstones date to the first half of the 13th century. Others, reused in constructions, are slightly older (before 1210). Their typology is homogeneous with diameters between 80 and $130 \mathrm{~cm}$. These millstones, often asymmetric, are characterised by dressing furrows and striations and show a high degree of wear and fragmentation. They are examples of an industrial application of hydraulic power and fall into the typological guidelines established by the first studies of medieval millstones.
\end{abstract}

Le corpus de meules provient d'un moulin à minerai hydraulique du XIII ${ }^{\mathrm{e}}$ siècle situé à Brandes-en-Oisans (France, Isère, Huez; MinvielLE LAROUSSE, à paraître). Elles ont été mises au jour en 2013 et 2014 dans le cadre d'une fouille programmée sur le quartier industriel de l'agglomération minière, active de la fin du XI ${ }^{\mathrm{e}}$ siècle à la première moitié du XIV ${ }^{\mathrm{e}}$ siècle (BAILLY-MAÎTRE, DUPRAZ, 1994). Le broyage est une étape de la chaîne opératoire des métaux polymétalliques (plomb, cuivre, argent) qui vise à enrichir le minerai, c'est-à-dire à le séparer de sa gangue, après l'extraction minière, et avant la phase métallurgique (fig. 1).

Notre étude intervient à la suite de plusieurs recherches. Après les premières descriptions de ces vestiges sur le plateau de Brandes (Héricart de Thury, 1807 ; MÜLler, 1899, 1901), le rôle des meules et moulins dans l'enrichissement du minerai a d'abord été questionné à l'échelle de sites (SERRES, 1995 ; Benoît, 1997, BaILly-MAître, 2002), avant d'aboutir à une première synthèse faite à partir des données de plusieurs exploitations minières contemporaines (BENOîT et alii, 1996). Pour apporter des éléments de réponses aux questions suscitées par cette synthèse, une étude typologique et technique systématique a été conduite sur les meules de Brandes réunies entre 1977 et 2009. Toutes avaient cependant été mises au jour en remploi, hors contexte de broyage (MINVIELLE LAROUSSE, BAILLYMAÎTRE, 2011a). La fouille du moulin a donc permis de constituer un corpus de meules nombreux, homogène, en contexte, et pleinement intégré dans la chaîne opératoire.

Les travaux comparables restent encore rares. Pour l'Antiquité une synthèse a été publiée, mais concernait des meules manuelles (Pulou et alii, 1997). Pour le Moyen Âge, seuls les corpus de Jihlava (République tchèque) et de Castel Minier (France, Ariège) ont été pour le moment approchés HRUBY, 2011 ; ARLES et alii, 2012). Les études du matériel de mouture issu de fouilles de moulins médiévaux à céréales sont aussi peu nombreuses. Si l'on considère les corpus de plus de vingt individus, nous n'avons pour seuls points de comparaison que celui issu de l'étude du village de Vilarnau (MARTZULFF et alii, 2008) et celui issu de la fouille du moulin de Thervay (JACCOTTEY, 2011b). 


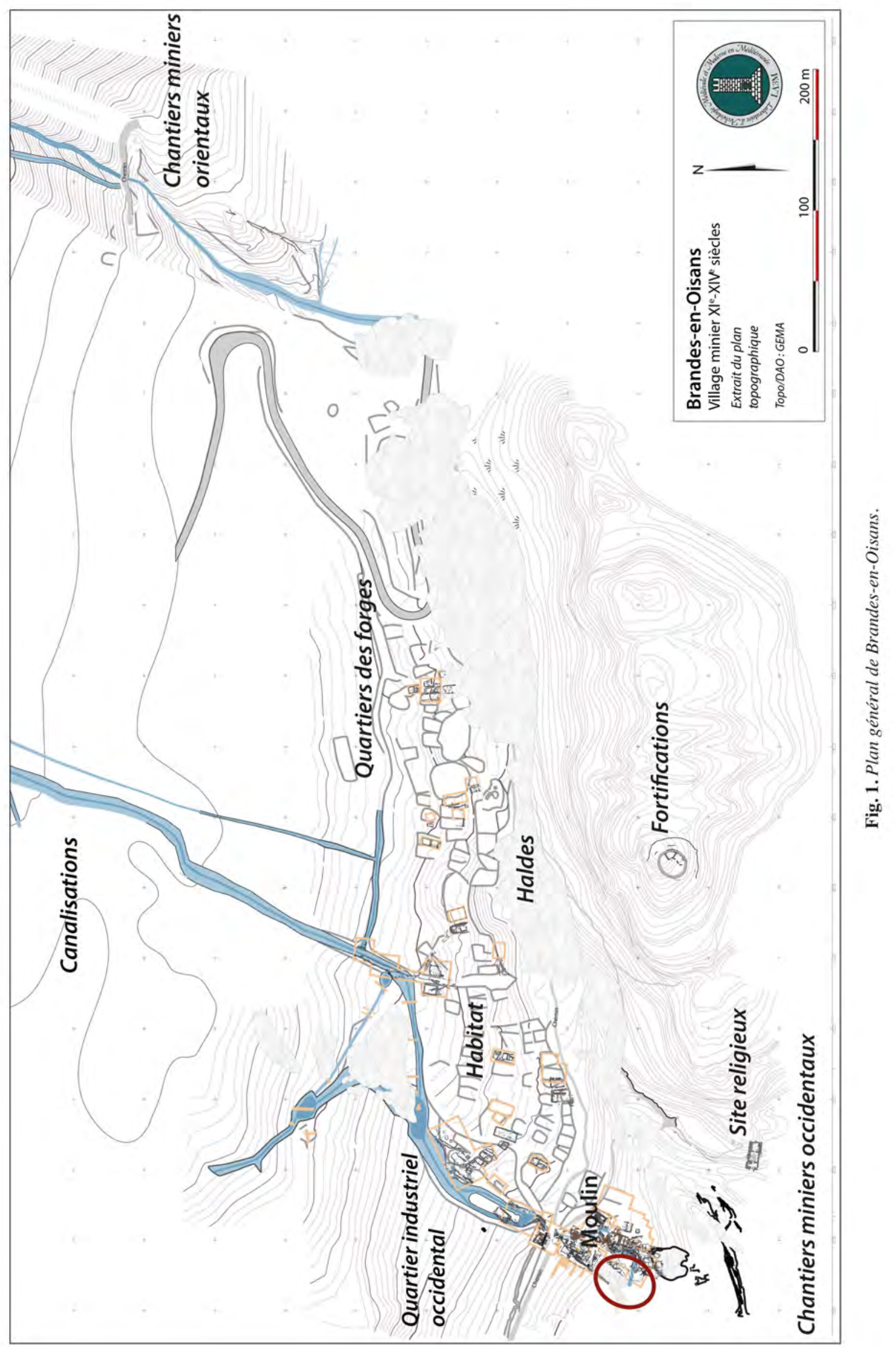


Devant l'indigence des recherches sur ce mobilier, nous proposons l'étude détaillée d'un lot de meules à minerai inscrit dans le XIII ${ }^{\mathrm{e}} \mathrm{s}$. Après avoir présenté brièvement le corpus et sa répartition spatiale par rapport au moulin, nous analyserons ses éléments morphométriques puis typologiques. Nous achèverons notre propos avec quelques réflexions sur le déroulement du broyage.

\section{PRÉSENTATION DU CORPUS}

\subsection{Composition}

Réuni au cours de deux campagnes successives, en 2013 et 2014, le corpus provient d'un secteur (B 121) situé entre les bras de la source Font-Morelle, au centre de la partie méridionale du quartier industriel. Il a livré 125 meules et fragments de meules ainsi que 309 éclats de meules.

Parmi les 125 individus, on compte notamment quatre meules complètes, cinq demi-meules, treize secteurs; le reste correspond à des fragments de tailles hétérométriques, allant de 5 à $50 \mathrm{~cm}$ de côté environ. Ce lot s'ajoute à un corpus accumulé depuis 1977 tout au long des opérations archéologiques. Il est composé de 83 meules et fragments de meules, de 119 éclats et de quatre ébauches. D'une façon générale, ces meules se caractérisent par leur fragmentation, ce qui limite les restitutions et les réflexions typologiques.

\subsection{Localisation et contexte}

Les meules ont été mises au jour dans un contexte de broyage du minerai. Elles se répartissaient dans un moulin et sur ses abords immédiats. Celui-ci fonctionnait avec une roue horizontale et était intégré dans le système hydraulique du quartier industriel. La canalisation le contournait au nord puis à l'ouest. Elle empruntait tout d'abord un ancien chantier minier, puis était bordée par des murs qui la séparaient de la chambre de broyage. Cette chambre, globalement quadrangulaire, était pourvue de deux étages. La partie basse contenait la roue, et à l'étage (non conservé), se situaient les meules. À son issue débutait un canal de fuite qui rejoignait quelques mètres plus en aval la canalisation. Le lien entre la canalisation et la roue s'effectuait par l'intermédiaire d'une gorgue $^{1}$, aménagée dans une faille du socle rocheux, en amont de la chambre de broyage (fig. 2).

1. Canal incliné en forme de pyramide tronquée renversée. Il passe au travers du mur du moulin pour acheminer l'eau du canal d'amenée à la roue horizontale. Il se termine par un canon de bois ou de métal (AMOURIC, 1984, p. 137-138).

\subsection{Datation}

Ce sont des meules du Moyen Âge central. Le terminus post quem global est situé dans la seconde moitié du $\mathrm{XI}^{\mathrm{e}}$ siècle, date des premiers travaux miniers sur le plateau. Le terminus ante quem global est placé dans la seconde moitié du XIV ${ }^{\mathrm{e}}$ s., moment où le village est majoritairement abandonné et où l'exploitation minière a cessé. Plus précisément, ces meules sont associées au moulin. Sa chronologie a été déterminée par plusieurs datations en dendrochronologie : construit à partir de 1210-1211, il était en fonction au moins jusqu'en 1227, date d'une réfection d'un poteau ${ }^{2}$. Mais, comme plusieurs meules étaient en réemploi dans le bâti du moulin, on suppose aussi une utilisation antérieure à 1211. La majorité des meules se place donc dans la première moitié du XIII ${ }^{\mathrm{e}} \mathrm{s}$., au moment où l'exploitation minière en général connaissait un apogée.

\subsection{Méthodologie}

Au cours de la fouille, chaque meule, fragment et éclat a été topographié et numéroté. En parallèle, chaque individu (hors éclat) a été enregistré dans des fiches papier, dessiné en plan sous forme de frottis, relevé en coupe, photographié et déterminé géologiquement. Enfin, les frottis ont été traités en négatif sous Photoshop, et les meules dessinées sous Illustrator selon les normes de dessins mises au point par le PCR «Évolution typologique et technique des meules...» (JACCOTTEY, FARGet, 2011 ; Minvielle LARousse, BAILLY-MAÎTRE, 2011b).

\subsection{Approvisionnement}

Le choix des meules est généralement déterminé par plusieurs facteurs : certains sont liés aux propriétés mécaniques de la roche (dureté, abrasion, ténacité, granulométrie, texture, rugosité); d'autres aux processus d'extraction (disponibilité, efficacité, ouvrabilité, durabilité); d'autres enfin à la distance à parcourir, qu'il soit question d'aller directement l'extraire ou de la faire venir. Il s'agit d'une balance entre les besoins fonctionnels, la connaissance comme la mâ̂trise technique, et la capacité d'investissement (FRONTEAU, BOYER, 2011).

À Brandes, les roches détritiques dominent: on trouve à $60,6 \%$ des conglomérats (77 individus); à 22,83\% des grès (vingt-neuf individus); et à 1,57\% des roches diverses (gneiss et granite pour deux individus). Enfin, dix-sept individus sont restés indéterminés, soit 13,39\%. Malgré tout, ils se rattachent soient à des grès, des conglomérats, des

2. Archéolabs - ARC13/R4021D/1; ARC14/R4095D. 


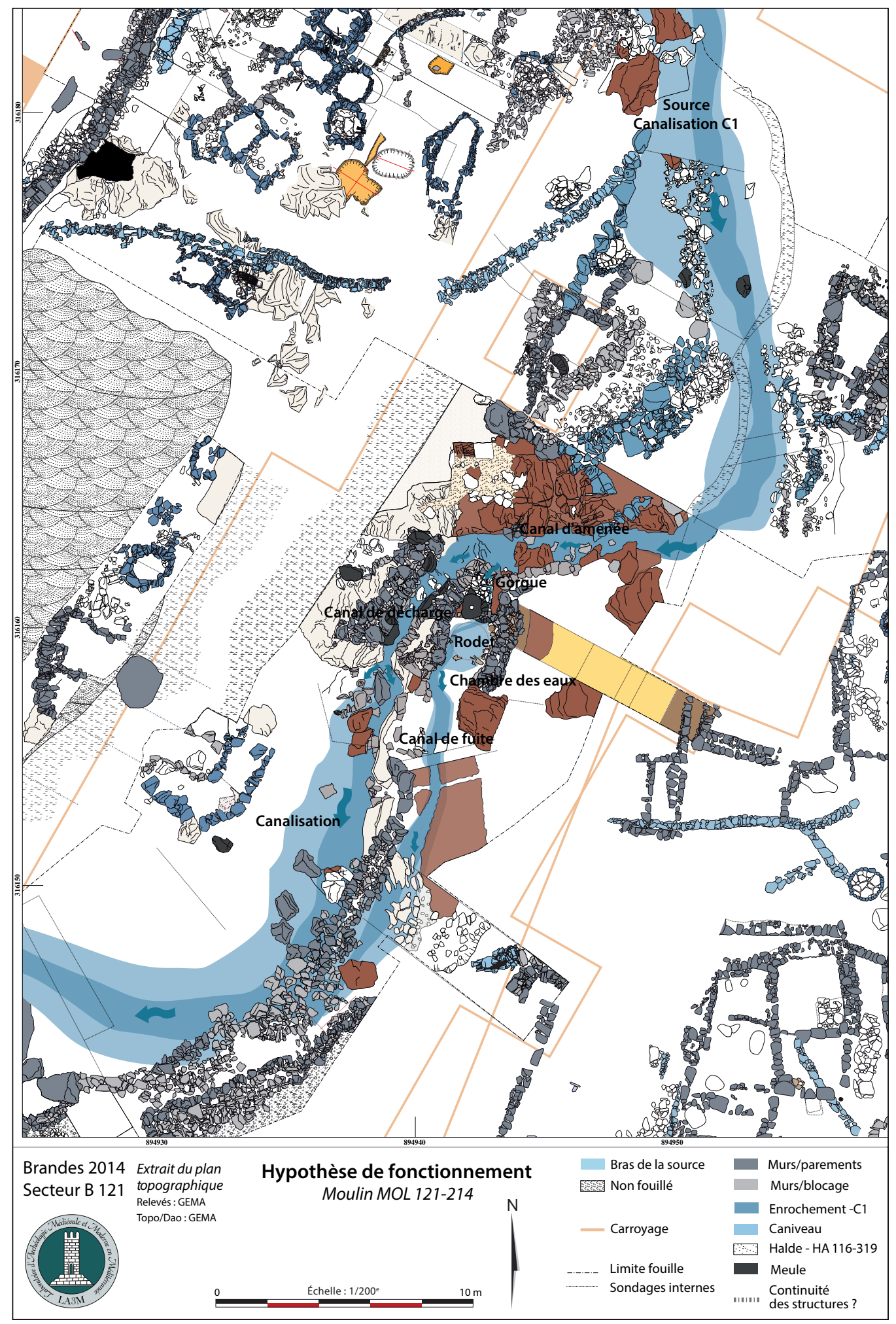

Fig. 2. Moulin de Brandes: hypothèse de fonctionnement. 


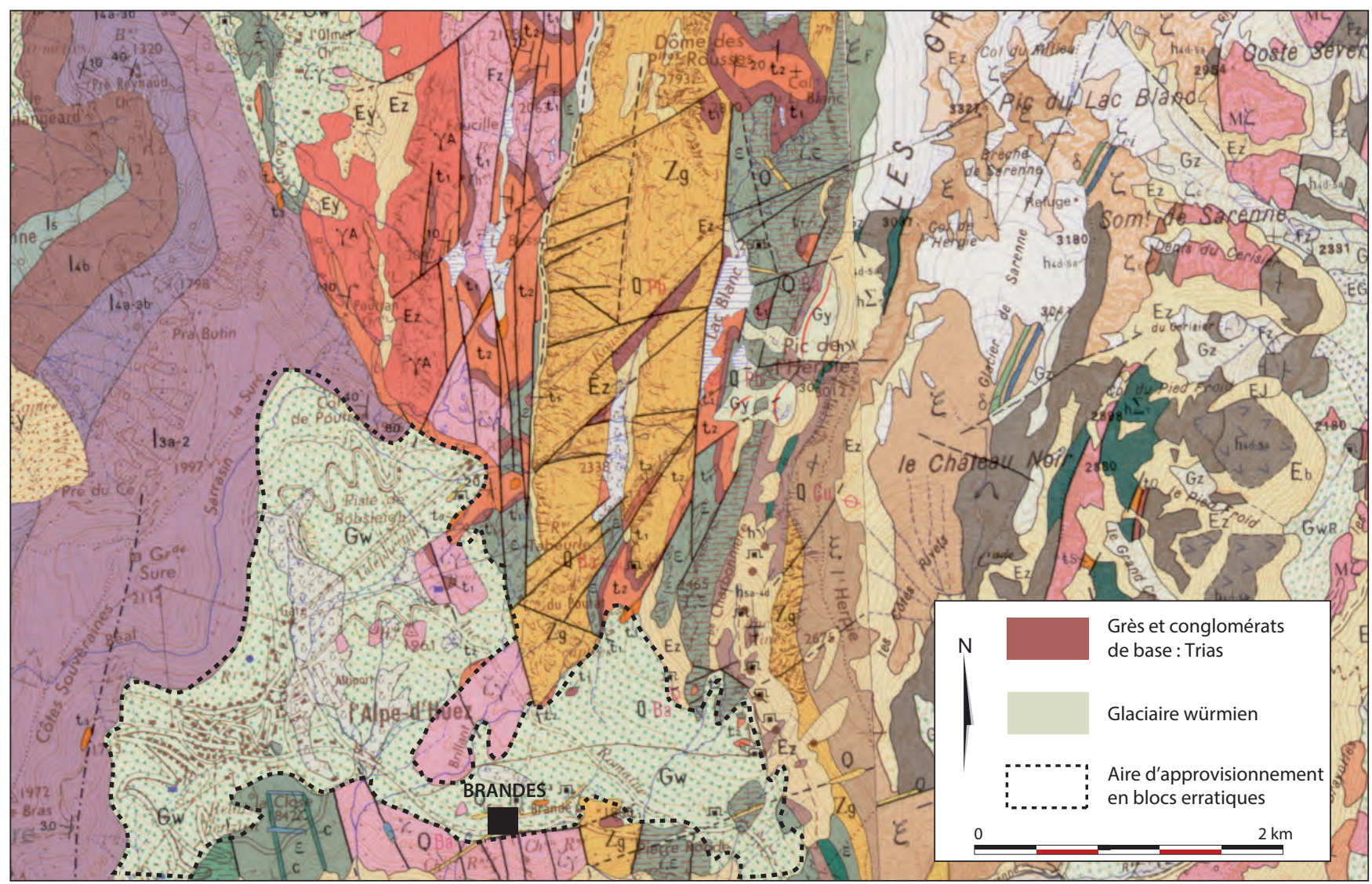

Fig. 3. Carte géologique et aire d'approvisionnement des meules (BRGM, Infoterre).

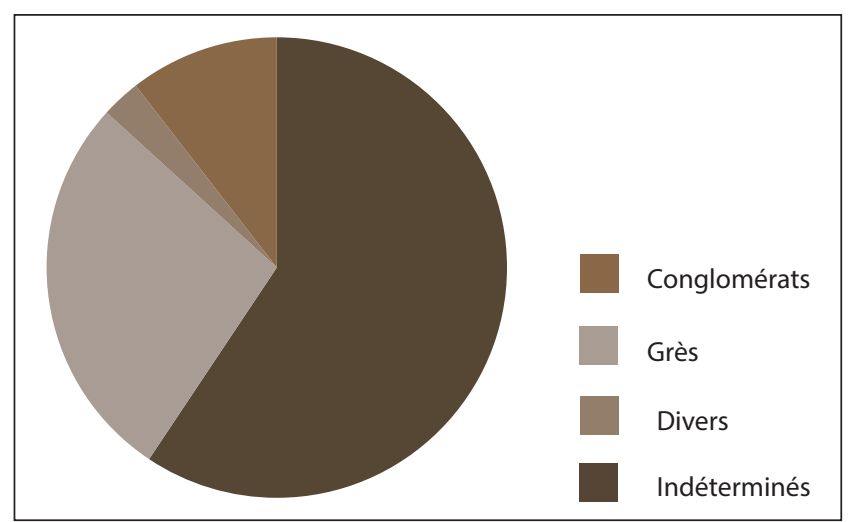

Fig. 4. Répartition des roches meulières.

gneiss ou des granites. Aucun autre type de roche n'a été aperçu (fig. 4$)^{3}$.

L'homogénéité des roches pose la question d'un éventuel choix de matériaux. Malgré nos recherches, nous n'avons pas remarqué d'ébauches ou de traces d'extractions

3. Réalisée par Stéphane Rouméjon (Docteur en Géologie, IPG), une typologie plus fine des grès et des conglomérats est disponible dans le rapport de fouille 2014. Nous avons repris la typologie formalisée en 2009, disponible dans la publication 2011. sur les quelques affleurements triasiques (grès conglomérats) situés en amont du site. La zone d'approvisionnement de l'agglomération de Brandes se situe ainsi majoritairement sur les moraines würmiennes, soit une aire d'un peu plus de $4 \mathrm{~km}$ de longueur et de $2 \mathrm{~km}$ de largeur (fig. 3). Quelques ébauches l'attestent encore, dont certaines en cours d'extraction directement sur des blocs erratiques. Il s'agit d'un périmètre d'extraction à la fois immédiat et attenant au site (Simonin, Jaccottey, 2013; Fronteau et alii, 2014). Ce type d'extraction à partir de blocs est courant dès la Protohistoire et a perduré jusqu' au XIX ${ }^{\mathrm{e}}$ siècle. Il s'oppose à l'exploitation du socle rocheux (ANDERSON, 2013).

Malgré cette solution qui peut sembler la plus simple, un choix a bien été opéré: les microconglomérats des blocs morainiques ont été préférés au gneiss du rocher SaintNicolas alors que celui-ci était disponible à l'extraction à quelques mètres des moulins. Ce sont soit les propriétés de la roche, soit les facilités d'extraction qui en sont à l'origine.

\section{RÉPARTITION DES MEULES}

Certes associés au moulin, de nombreux fragments étaient aussi contenus dans les colluvions et remblais postérieurs à son abandon, bien souvent d'une composition identique à ceux déposés lors de son fonctionnement. 


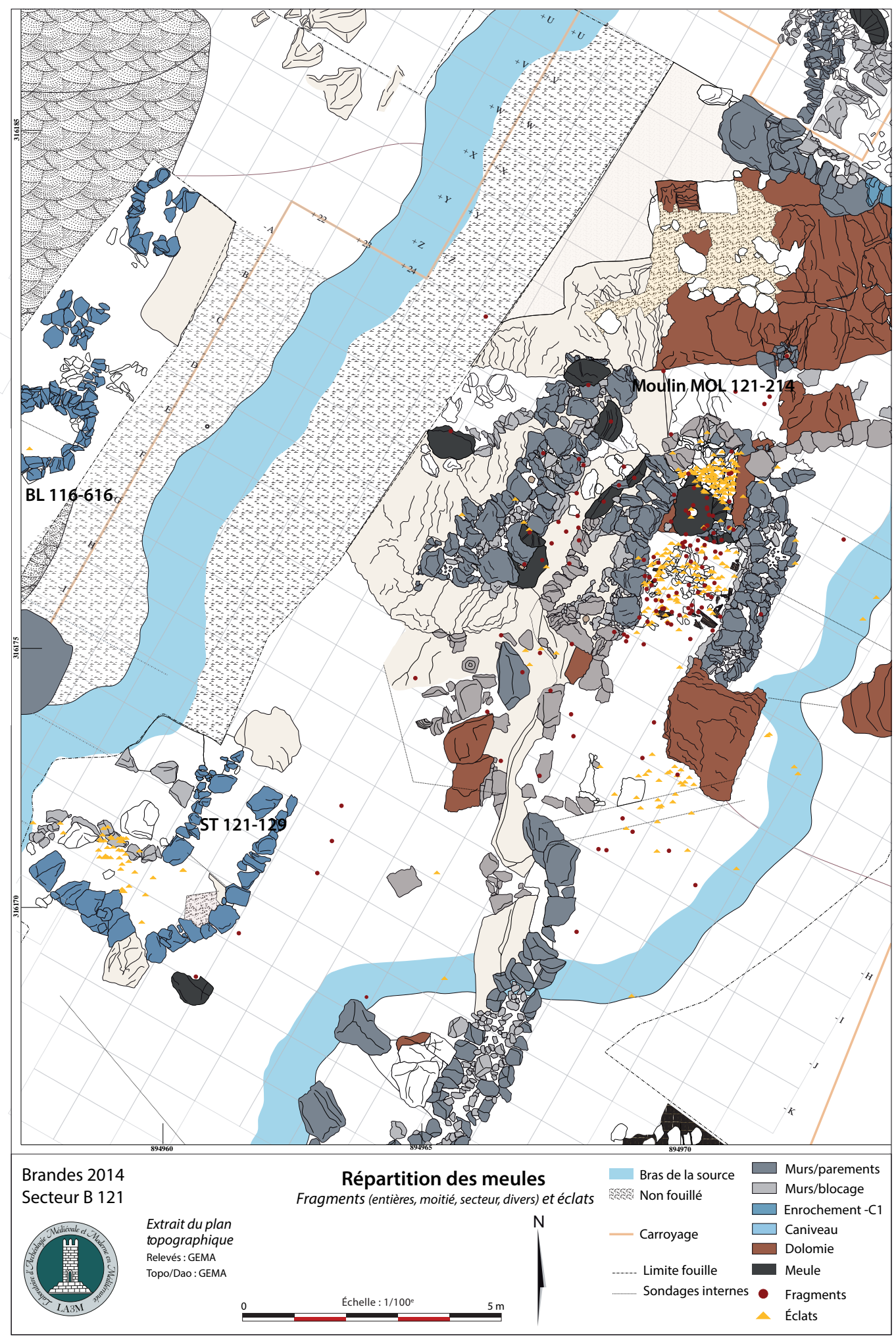

Fig. 5. Localisation des meules autour du moulin. 


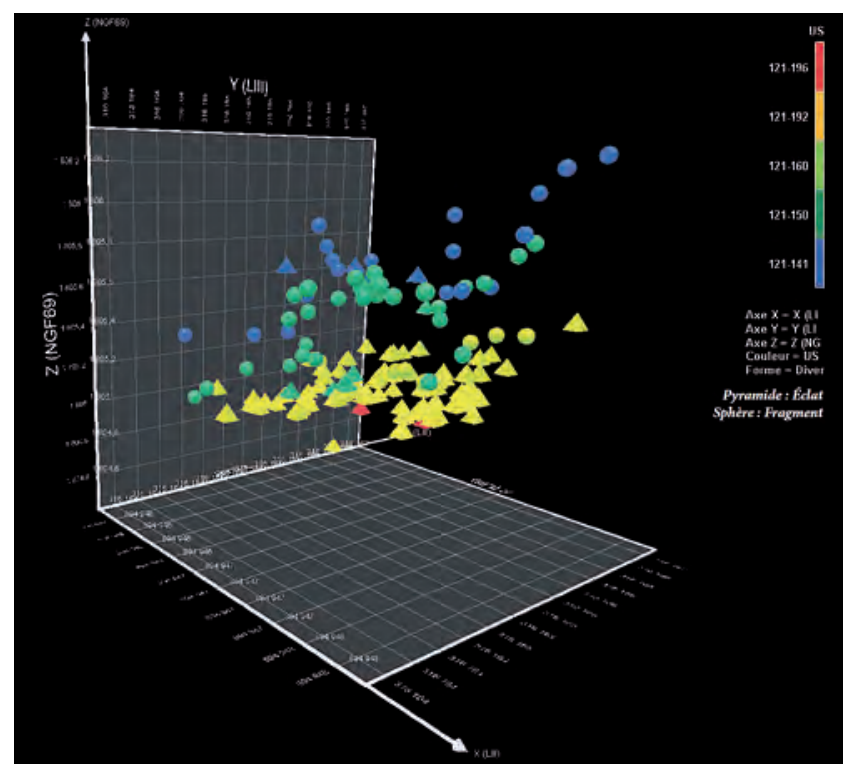

Fig. 6. Répartition des fragments et éclats de meules dans la chambre de broyage.

La difficulté était donc de les discriminer, le tout dans une stratigraphie en perpetuum mobile car circulation hydraulique avait perduré dans ce secteur jusqu'à nos jours (source Font-Morelle).

\subsection{Répartition stratigraphique}

Meules et fragments de meules étaient contenus dans quatre types de contexte. Dix-neuf d'entre eux étaient tout d'abord réemployés dans le bâti. Cela concerne le mur de fond de la chambre de broyage, le mur ouest du canal de dérivation, et le mur est du moulin. Ensuite, on compte quarante-quatre individus répartis dans les remplissages de la chambre de broyage et onze individus contenus dans les divers remblais déversés dans le canal de fuite du moulin. Enfin, les colluvions issues de l'abandon de la canalisation $\mathrm{C} 1$ contenaient vingt-huit individus. Le lien de ces derniers avec le moulin est peut-être moins évident. Le reste du corpus était dispersé dans plusieurs unités plus ponctuelles, ou liées à la sédimentation moderne et contemporaine du site.

Les éclats de meule avaient une répartition plus homogène avec une première concentration située au sommet de la gorgue du moulin. Une seconde s'observe dans la chambre de broyage, au sein des premières (en chronologie) unités de comblement, probablement proches du fonctionnement. Enfin, une troisième se situait dans le comblement principal d'une structure de lavage située légèrement en contrebas [ST 121-129] et une dernière dans un bassin de lavage implanté quelques mètres plus à l'ouest [BL 116616].

\subsection{Interprétations}

La chambre de broyage contenait l'immense majorité des meules, ce qui nous a d'ailleurs permis de corroborer son identification. Si la partie amont du moulin est pauvre, ce n'est pas le cas de la partie aval sur laquelle se répartissait une quinzaine de fragments, et même quelques meules entières. On peut tout simplement lier cette différence au sens du courant qui favorisait le charriage de fragments en aval. Une dizaine de fragments était enfin échouée dans le canal de dérivation, au travers duquel les eaux de la canalisation passaient après abandon.

La distinction moulin/abords se marque davantage avec la répartition des éclats. Sauf quelques exceptions, ils ont tous été mis au jour dans la chambre de broyage dans des espaces hors eau, ou du moins protégés du flux. Ainsi au sommet de la gorgue, qui était entièrement au sec, et à la base de la chambre des eaux. Cette dernière concentration s'explique moins facilement, sauf si la roue était surélevée (fig. 6). Le plan suggère en outre que ces éclats pourraient être répartis en anneau. Faut-il y voir l'emplacement des meules en négatif, autour desquelles les éclats se seraient accumulés?

\section{3. ÉLÉMENTS MORPHOMÉTRIQUES}

La morphométrie est le principal ensemble de caractères distinctifs des meules, en particulier pour la période médiévale. La taille peut l'inscrire dans le temps, la courbure de la surface active lui donne sa fonction.

\subsection{Le diamètre}

\subsubsection{Le calcul du diamètre: méthodes et biais}

Il existe plusieurs méthodes pour calculer le centre d'un cercle à partir d'un fragment de circonférence. Celle que nous utilisons provient de la géométrie d'Euclide: toutes les médiatrices des cordes d'un cercle se rencontrent en son centre (Livre III, propriété 25). Cette méthode est valable pour calculer aussi bien le diamètre de la meule que celui de l'œil. Elle nécessite cependant deux précautions : la première est de bien avoir des points se trouvant sur la circonférence effective du cercle, et non sur un arrondi tronqué. La seconde est de tracer des cordes les plus longues possible pour minimiser l'erreur. Les deux biais énoncés sont à l'origine de la plupart des erreurs de diamètre lorsque l'on est confronté à des fragments soit petits, soit pourvus d'un bord irrégulier. La marge d'incertitude peut être de 1 à plus de $10 \mathrm{~cm}$.

Avec ce corpus où les bords réguliers sont loin d'être majoritaires, on s'est servi dans certains cas des dernières stries situées sur les feuillures. Celles-ci rendent compte 


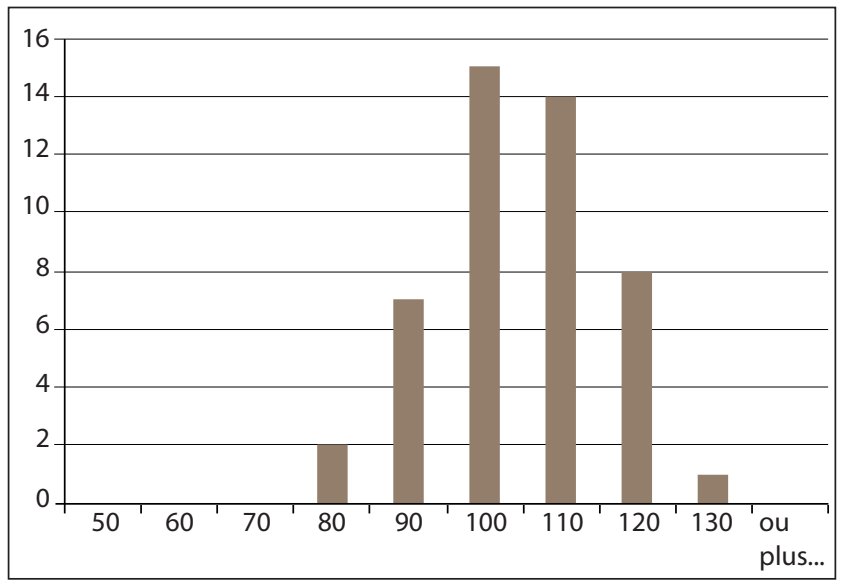

Fig. 7. Diamètres estimés des meules (2013-2014).

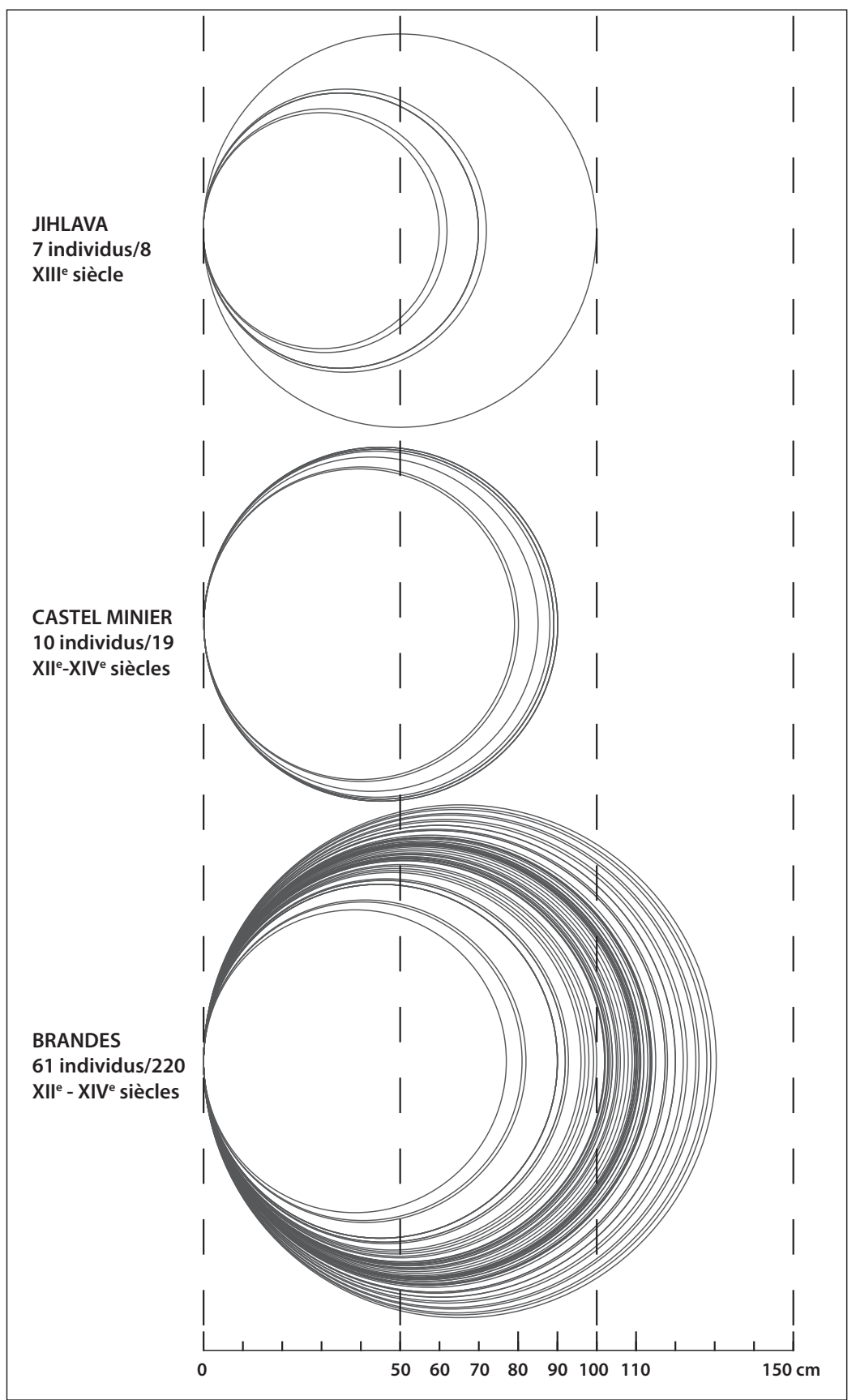

Fig. 8. Diamètres estimés des corpus de Brandes, Castel-Minier et Jihlava. 
des rotations, donc du diamètre des surfaces actives. Elles donnent un diamètre minimum. Lorsque la meule se prolonge encore quelques centimètres au-delà de la surface active, nous avons estimé un diamètre maximum. Dans tous les cas, ce calcul n'a été pratiqué que sur des fragments assez expressifs ; lorsqu'il y avait un doute sur le tracé des cordes, le diamètre a été laissé indéterminé. Le calcul des diamètres en effet est une opération sensible: c'est l'un des points de comparaison entre les corpus, et l'un des marqueurs de l'évolution chronologique des meules. Pour compenser ces biais, on regardera tout particulièrement les mesures les plus fiables: prises sur les meules entières, les demi-meules, et les secteurs (vingt-deux individus sur 125).

\subsubsection{Les diamètres du corpus 2013/2014}

On a pu déterminer le diamètre de quarante-huit individus sur 125 . Les meules ont des diamètres allant de 80 à $130 \mathrm{~cm}$, soit un écart non négligeable de $50 \mathrm{~cm}$. On observe une très nette prédominance des meules comprises dans les classes de 100 et $110 \mathrm{~cm}$ (respectivement quinze et quatorze individus). Les classes de 90 et $120 \mathrm{~cm}$ sont des marges équivalentes avec sept et huit individus. En revanche, les classes de 80 et $130 \mathrm{~cm}$ sont quasiment vides, seulement deux et un individu. Si l'on prend les mesures les plus fiables, avec les meules entières, en demi ou en secteur, on arrive à une répartition presque exclusivement située entre 100 et $120 \mathrm{~cm}$ (fig. 7).

Malgré l'homogénéité du corpus, il convient de poser la question de l'évolution chronologique. On peut différencier en relatif les meules utilisées dans les structures du moulin antérieures à son fonctionnement; celles empilées dans la chambre de broyage, proches de la phase ultime de son fonctionnement, et celles contenues dans les différentes unités de recouvrement. Or, nous ne percevons pas de tendance, les diamètres restent globalement identiques et correspondent de surcroît à ceux des meules étudiées en 2009 (treize individus avaient été déterminés). On avait des meules allant de 77 à $112 \mathrm{~cm}$, avec une majorité d'entre elles comprise entre 100 et $110 \mathrm{~cm}$. Ainsi, on aurait plutôt une relative homogénéité des diamètres entre le XII ${ }^{\mathrm{e}}$ et le $\mathrm{XIV}^{\mathrm{e}} \mathrm{s}$. à Brandes.

\subsubsection{Comparaisons et contextualisation}

Les diamètres des meules de Brandes sont en revanche légèrement supérieurs à ceux qui ont été déterminés sur d'autres exploitations minières. À Castel Minier (Ariège) le diamètre est compris entre 80 et $90 \mathrm{~cm}$ pour un corpus de dix-neuf individus daté des XIII ${ }^{\mathrm{e}}-\mathrm{XIV}^{\mathrm{e}} \mathrm{s}$. Les meules sont plus petites à Jihlava (République tchèque) avec un diamètre allant de 60 à $70 \mathrm{~cm}$, malgré une exception à $100 \mathrm{~cm}$. Daté $\mathrm{du} \mathrm{XIII}^{\mathrm{e}}$ s., ce corpus comprend huit individus. Nous ne possédons enfin que quelques rares informations sur les meules de Pampailly (Rhône). Leur diamètre serait compris dans une fourchette de 60 à $90 \mathrm{~cm}$ pour un corpus non daté précisément (contemporain ou antérieur aux $\mathrm{XIV}^{\mathrm{e}}-\mathrm{XV}^{\mathrm{e}} \mathrm{s}$. semblet-il). En prenant en compte l'ensemble de ces données, on voit se dessiner des meules de 60 à $130 \mathrm{~cm}$, toutes datées des $\mathrm{XII}^{\mathrm{e}}-\mathrm{XIV}^{\mathrm{e}} \mathrm{s}$. On observe donc de fortes disparités dans les diamètres des meules utilisées dans des exploitations pourtant contemporaines (fig. 8).

Au-delà de ces comparaisons minières, les diamètres des meules à minerai semblent en majorité inférieurs aux diamètres connus pour les meules à blé, à période égale. D'après un récent état de la question construit à partir de 233 meules issues de cinquante-quatre sites, une augmentation progressive du diamètre des meules a été constatée entre le $\mathrm{V}^{\mathrm{e}}$ et le $\mathrm{XVI}^{\mathrm{e}}$ siècle. Aux XIII ${ }^{\mathrm{e}}$ et XIV ${ }^{\mathrm{e}} \mathrm{s}$., le diamètre moyen des meules à blé est de 126,5 cm (BELMONT et alii, 2016). Si les meules de Brandes sont parfois proches de cette moyenne, celles de Castel Minier et de Jihlava en sont loin. Les meules à minerai mettent par conséquent en évidence des diamètres hétérométriques dans une période relativement courte. Cette diversité transparaît moins pour le moment dans les diamètres des meules à blé; mais comme les études typologiques des meules médiévales en sont à leur début, il faut que les individus datés soient plus nombreux pour que les tendances s'affinent et soient statistiquement viables.

\subsection{L'épaisseur}

Du plan, venons-en à la coupe. Les fragments de meules sont épais de 3 à $31 \mathrm{~cm}$. La plupart (80 individus sur 117) sont situés dans une fourchette de 5 à $15 \mathrm{~cm}$. Une partie plus faible se situe entre 15 et $25 \mathrm{~cm}$ (vingt-sept individus). Les sept individus se situant entre 1 et $5 \mathrm{~cm}$, comme les quatre individus pourvus d'une épaisseur de plus de $25 \mathrm{~cm}$ sont minoritaires (fig. 9). L'épaisseur des meules entières,

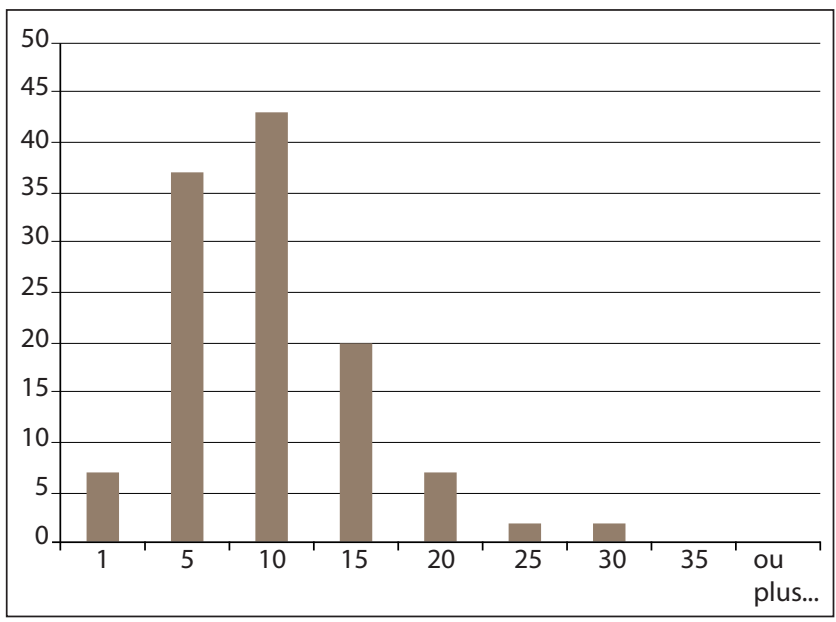

Fig. 9. Répartition des épaisseurs maximales des meules (corpus 2013-2014). 


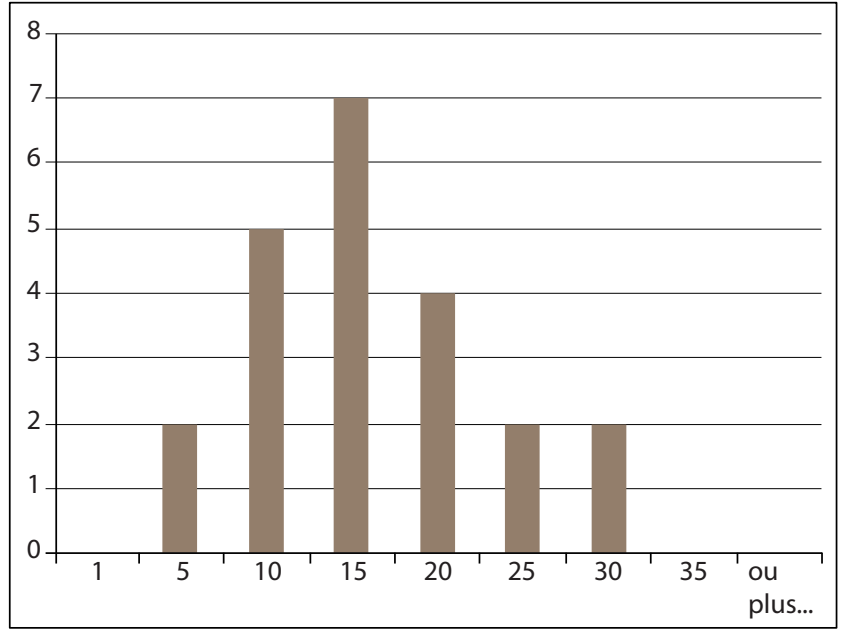

Fig. 10. Répartition des épaisseurs maximales des meules entières, moitié et secteur (corpus 2013-2014).

en moitié ou en secteur nuance quelque peu cette première répartition. On note toujours une concentration globale des individus entre 10 et $20 \mathrm{~cm}$, mais au contraire du corpus complet, la fourchette $15-20 \mathrm{~cm}$ est plus importante. Cette épaisseur semble mieux convenir pour donner une moyenne au corpus (fig. 10). En effet, les fragments sont majoritairement des feuillures, ce qui tire artificiellement vers le bas les données.

L'épaisseur est une donnée majoritairement tronquée. Comme la totalité des meules mise au jour est en contexte de rejet ou d'abandon après fonctionnement, nous ne mesurons que l'épaisseur post-broyage; celle-ci diffère de plusieurs dizaines de centimètres de l'épaisseur initiale, donnée après le façonnage. Par ailleurs, on retrouve le problème de la fragmentation des meules. Mesurer une épaisseur représentative de la meule sur un fragment de quelques centimètres de large est dans bien des cas impossible. Enfin, l'épaisseur dépend de la position du fragment: une feuillure sera ainsi plus fine qu'un entrepied. Les données présentées sont par conséquent tributaires de ces trois biais. Ici encore, il faut se tourner vers les meules entières, les demi-meules ou les secteurs pour obtenir les mesures les plus représentatives.

\subsection{Les flancs et surfaces opposées}

La forme des flancs et des surfaces opposées est moins standardisée. Seuls 12 bords ont été reconnus parmi le corpus. Leurs flancs sont subverticaux (2014-175) ou obliques (2014-174). On ne perçoit pas de mise en forme particulière ni de traces d'aménagements (ferrures, outils...), mais parfois des traces de taille sur les surfaces opposées (2013-043, 2014-103, 2014-119, 2014-121). Leur traitement est toutefois réduit au minimum, ces surfaces restent très grossières. Les meules dormantes sont les plus frustes. En dehors de la surface active, on est en présence d'un bloc brut dissymétrique à peine mis en forme.

\subsection{Meules dormantes, meules tournantes}

Trois éléments distinguent une meule dormante d'une tournante. Il s'agit en premier lieu de la présence ou non d'une encoche pour l'anille. En second lieu, la forme de la surface active est généralement caractéristique: concave pour la tournante, convexe pour la dormante. En dernier lieu, mais plus discutable, il s'agit de l'épaisseur des meules, traditionnellement plus élevée pour une dormante; mais seulement avant utilisation. Après le rejet, les deux peuvent être d'une épaisseur équivalente.

Toujours en raison de la fragmentation du corpus, distinguer une meule dormante d'une meule tournante a été très malaisé. Sur 125 individus, nous n'avons pu en déterminer de façon certaine que vingt-cinq. Il y a au moins onze meules dormantes et quatorze meules tournantes. Cette forte proportion d'indéterminés implique que nous ne pouvons pas interpréter cette donnée. Si l'on prend uniquement les meules contenues dans la chambre des eaux, on se heurte au même problème: trois dormantes, deux tournantes, et quatre-vingt-quatre indéterminées.

Il est possible que le nombre de tournantes soit au final plus important que les dormantes. C'est en tout cas ce que l'étude des meules du moulin de Thervay a mis en évidence, et que la différence d'épaisseur au départ entre les deux corrobore. On utilise plusieurs meules tournantes successives, sur une meule dormante. Nous ne pouvons cependant pas vérifier ou réfuter cette généralité avec ce corpus.

\section{4. ÉLÉMENTS TYPOLOGIQUES}

Les meules hydrauliques médiévales semblent assez homogènes typologiquement (BELMONT et alii, 2016). Ici, nous sommes en présence d'un corpus typologiquement uniforme pour les œils, et les anilles. En revanche, l'habillage varie d'une meule à l'autre; et elles peuvent enfin se distinguer entre des individus monolithes et d'autres, assemblés en carreaux.

\section{1. $L$ 'oil}

L'œil, ou œillard perfore entièrement les meules tournantes et dormantes. Nous avons pu réunir trentesept individus pourvus d'un œil, le plus souvent partiel. Systématiquement circulaire, il permet à l'arbre moteur de traverser la meule dormante pour rejoindre l'anille fixée dans la meule tournante. Par ailleurs, il est également utilisé pour introduire le minerai dans les meules, à partir de la tournante. Outre sa forme, ce composant a deux caractéristiques: son diamètre et son épaisseur.

Le diamètre est déterminé par ces deux fonctions, et en même temps contraint par la nécessité de ne pas trop rogner la surface active. On trouve sur le corpus 2013/2014 des 
œils avec un diamètre allant de 14 à $21 \mathrm{~cm}$. La très grande majorité se situe entre 15 et $20 \mathrm{~cm}$. Le diamètre est globalement constant, une partie des variations est probablement la conséquence des imprécisions des calculs de diamètre. Cela étant, le diamètre des meules variant, la question du rapport des deux diamètres se pose. Le ratio diamètre œil/ diamètre meule a été fait chaque fois que c'était possible.

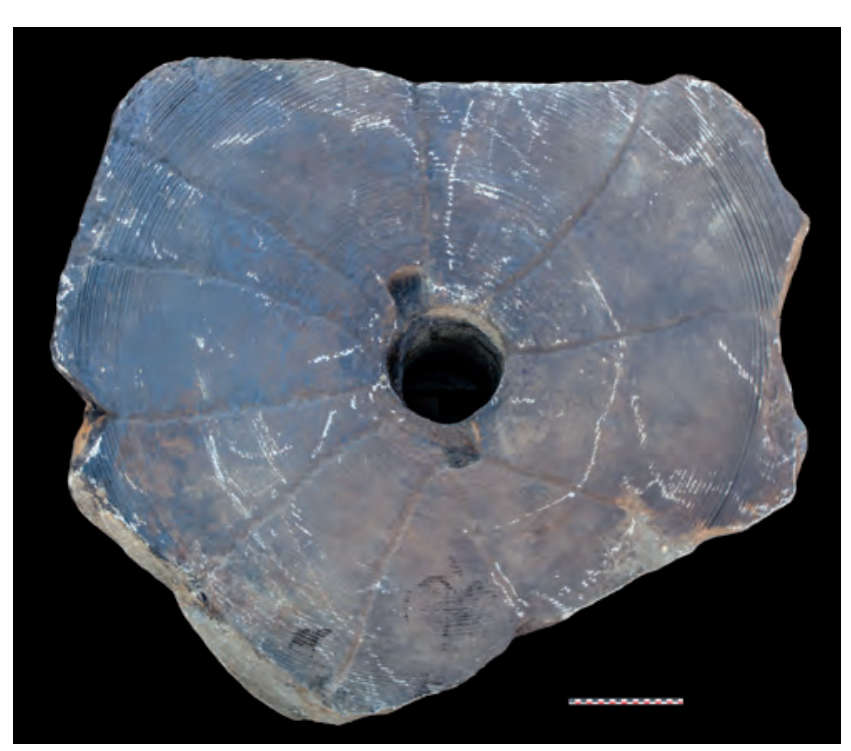

Fig. 11. Meule 2014-174. Rayonnage continu et décalage des centres de l'anille et de l'œil.
Il ressort déjà que l'œil est généralement 6 à 7 fois plus petit que le diamètre de la meule. Mais, nous n'observons pas de corrélation entre les deux données. Si en général, l'œil reste fonction de la taille de la meule, dans les détails, il serait taillé en fonction du mécanisme (diamètre de l'arbre, taille de l'anille), et peu importeraient les variations du diamètre des meules.

\subsection{L'anille}

L'anille ou le «gros fer» est la pièce métallique qui permet la transmission du mouvement rotatif de l'arbre moteur à la meule tournante. Il s'agit d'une pièce de fer qui peut être de forme variable, encastrée dans une encoche taillée dans la surface inférieure de la meule tournante. Pièce spécifique au moulin, elle est le plus souvent récupérée et réutilisée. Il est extrêmement rare d'en retrouver en fouille: nous n'en avons pas mis au jour un seul exemplaire, ni même de fragments. Par conséquent, nous ne pouvons l'approcher que par l'intermédiaire de son encoche.

Parmi les 125 fragments du corpus, seuls sept d'entre eux étaient pourvus d'une encoche d'anille, complète ou partielle. Ces encoches sont toutes de forme identique: grossièrement quadrangulaires, elles sont toujours légèrement dissymétriques (fig. 11 et 12). Le grand côté, perpendiculaire au bord de l'œil, mesure de 27 à $30 \mathrm{~cm}$, le petit côté, parallèle au bord de l'œil, de 5 à $6 \mathrm{~cm}$. Lors de

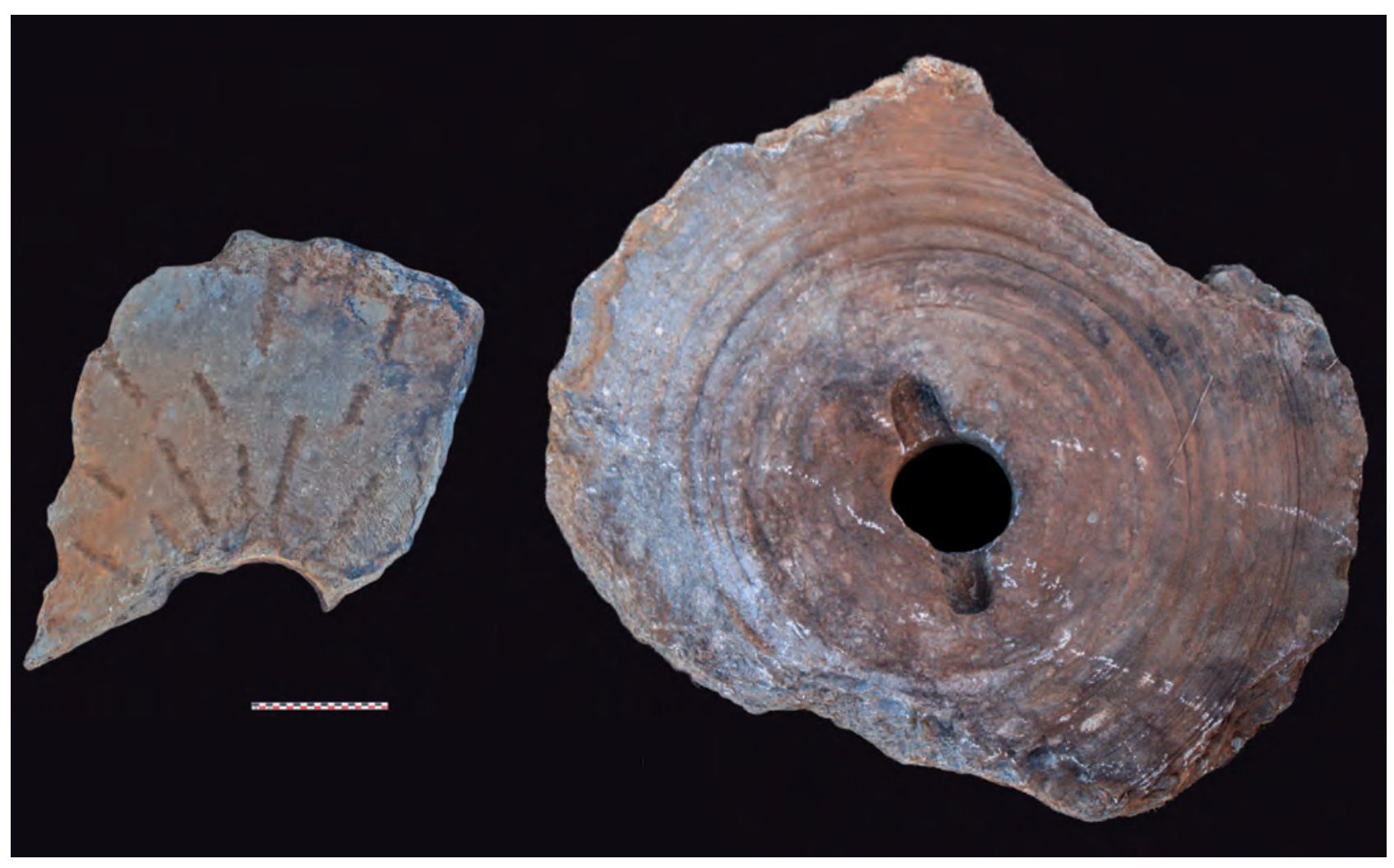

Fig. 12. Meule 2014-039 (à gauche): rayonnage discontinu. Meule 2013-196 (à droite): meule non rayonnée. 


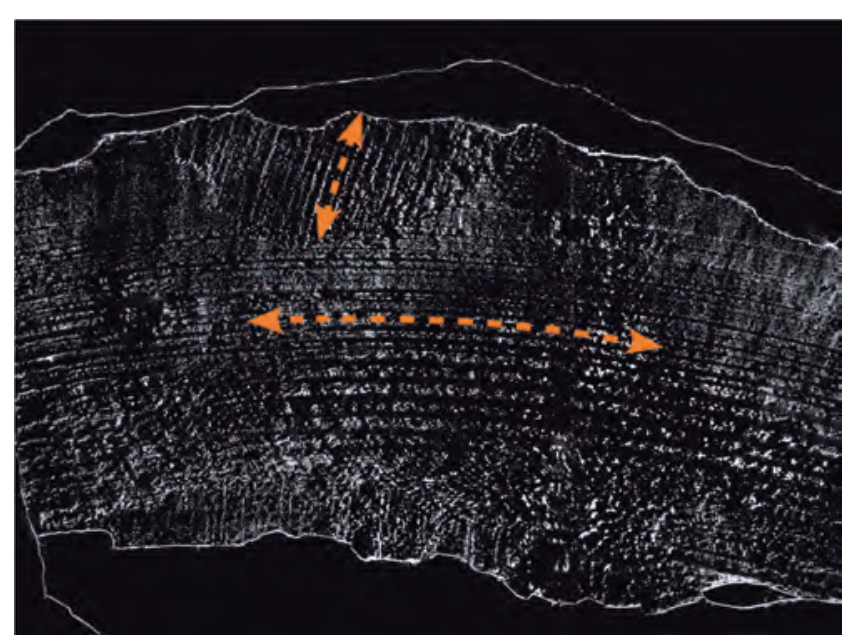

Fig. 13. Meule 2013-041. Deux sens de rotation visibles par les stries.

l'abandon de la meule, l'encoche est épaisse de 2,5 à $4 \mathrm{~cm}$. Lorsque l'on observe les trois meules complètes pourvues d'une anille (2013-196, 2014-163, 2014-174), on remarque que l'encoche n'est pas taillée au centre de l'œil, mais décalée de 1,5 à $2 \mathrm{~cm}$ environ. Est-ce pour ménager un espace plus important qui facilitait le versement du minerai?

\subsection{Le rayonnage}

Le rayonnage est fréquent: quarante-sept individus sur 129 l'étaient, qu'ils appartiennent à des meules dormantes ou à des tournantes. La plupart des meules n'avaient conservé qu'un rayon empêchant toute classification. Cela étant, l'apport de nouveaux individus et la prise en compte d'autres corpus (Castel-Minier, Jihlava) conduisent à nuancer la typologie construite en 2009. En effet, nous remarquons une plus grande variété dans les rayonnages. L'impression actuelle est une forme d'opportunisme sur leur répartition sur les surfaces actives. La seule constante serait une couverture aléatoire de la surface, à la condition qu'elle soit homogène. Le nombre de rayons n'est donc plus un critère. Il semble que seuls les rayonnages continus, discontinus et mixtes puissent former trois catégories distinctes, encore faut-il avoir un fragment assez important pour être en mesure de les déterminer (fig. 11 et 12).

Quoi qu'il en soit, le rayonnage est une caractéristique au Moyen Âge des meules à minerai. Cette forme d'habillage des surfaces actives était pourtant courante durant l'Antiquité pour les meules à blé et s'est diffusée à nouveau au cours de la période moderne. Quelques exceptions pouvaient néanmoins exister, comme le laisse supposer une illustration des XIV ${ }^{\mathrm{e}} \mathrm{XV}^{\mathrm{e}}$ s. (LEPAREUX-COUTURIER et alii, 2011).

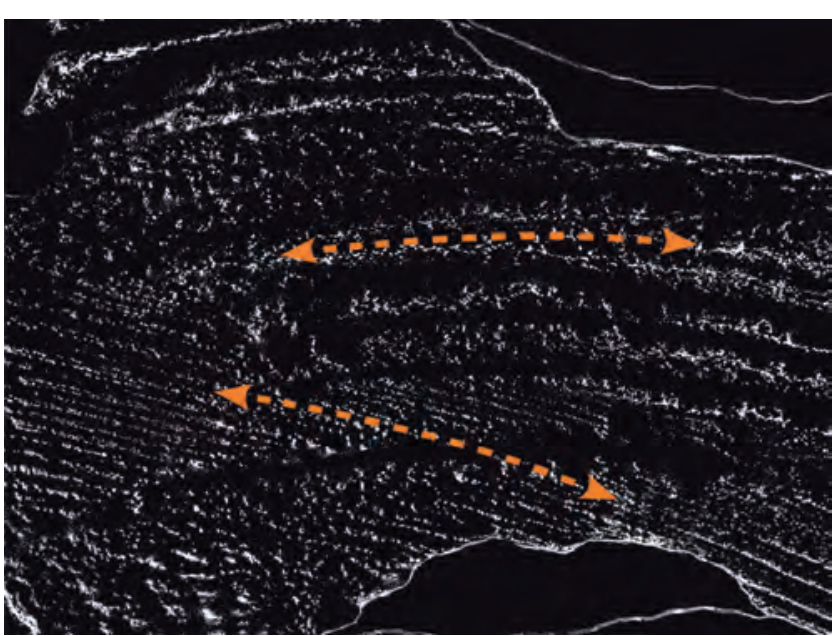

Fig. 14. Meule 2013-049. Deux sens de rotation visibles par les stries.

\subsection{Des meules assemblées}

En analysant les stries qui marquaient les surfaces actives des meules, nous avons observé à plusieurs reprises plusieurs sens de rotation divergeants. Certains avaient un léger décalage, mais d'autres étaient opposés. Nous avions alors supposé la présence de meules assemblées en carreaux, probablement irréguliers, aux côtés de meules monolithes plus classiques. Trois fragments issus du moulin étaient pourvus de deux sens de rotation, mis en évidence par les stries (2013-057, 2013-041, 2013-049), ils s'ajoutent à deux autres observés en 2009 (2007-0-1507 et 2007-0-1504).

Le fragment 2013-057 est pourvu sur sa partie distale de stries de type feuillure, qui ont été recoupées par des stries de type entrepied avec un autre sens de rotation. Il semble avoir été disposé sur une autre partie de la meule, ce qui expliquerait la transition brusque entre l'épaisseur des deux types de stries. D'autre part, il a légèrement pivoté, ce qui explique la double rotation.

Le fragment 2013-041 est encore plus expressif (fig. 13). Ses stries sont de type feuillure, mais on remarque deux sens de rotation. Il y a d'abord la partie centrale de la surface active, et ensuite deux fragments restreints d'une ancienne surface active situés au-dessus et au-dessous. Ici, la rotation du fragment a été de l'ordre de $90^{\circ}$.

Le fragment 2013-049 montre aussi deux utilisations successives (fig. 14). Cette évolution se caractérise par un changement du centre de rotation de la meule, ce qui a engendré les deux sens visibles au niveau des stries. Comme l'on passe également de stries de type entrepied à des stries de type feuillure, le fragment a aussi changé de place dans la meule.

Le fragment 2007-0-1507 est pourvu de trois surfaces actives successives. Les deux premières occupent la majeure partie de sa surface et la troisième, plus petite, 


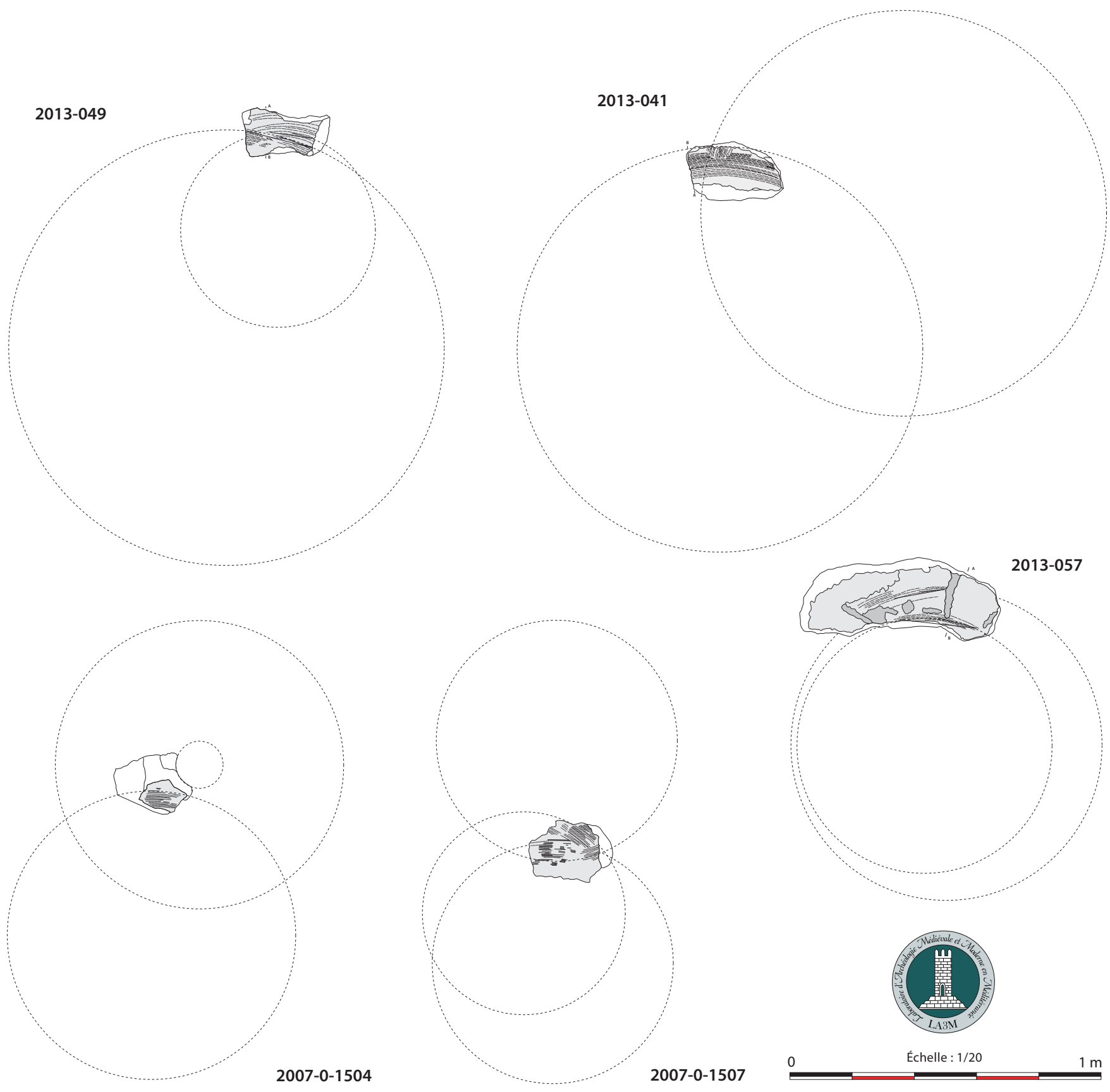

Fig. 15. Meules assemblées.

se situe à droite du fragment. Autant les deux premières ne témoignent que d'un déplacement modeste du fragment dans la meule, autant la troisième montre un retournement à près de $180^{\circ}$.

Le fragment 2007-1504 enfin est celui qui est peut-être le plus évident. Il prend dans la meule deux places complètement différentes et incompatibles entre elles. La première utilisation fonctionnait avec l'œil, et la seconde avec la surface striée, de type feuillure.

L'hypothèse de voir des meules assemblées reste encore fragile, car nous n'expliquons pas pourquoi leur nombre n'est pas plus important et pourquoi nous n'avons aucun indice lié au système de cerclage (fig. 15). Jusqu'aux années 1980, on n'envisageait d'ailleurs pas l'existence de ce type de meule pour le Moyen Âge (BRUGGEMANN, 2003 , p. 232). Depuis, quelques mentions ont été repérées dans les textes modernes, et une seulement dans un texte de 1452 (BELMONT, 2003, p. 282). Les meules mises au jour à Brandes reculeraient encore l'utilisation de cette technique aux XII ${ }^{\mathrm{e}}$ et XIII ${ }^{\mathrm{e}}$ s., soit 1400 ans après l'expérience du moulin délien (BRUNET, 1997). 


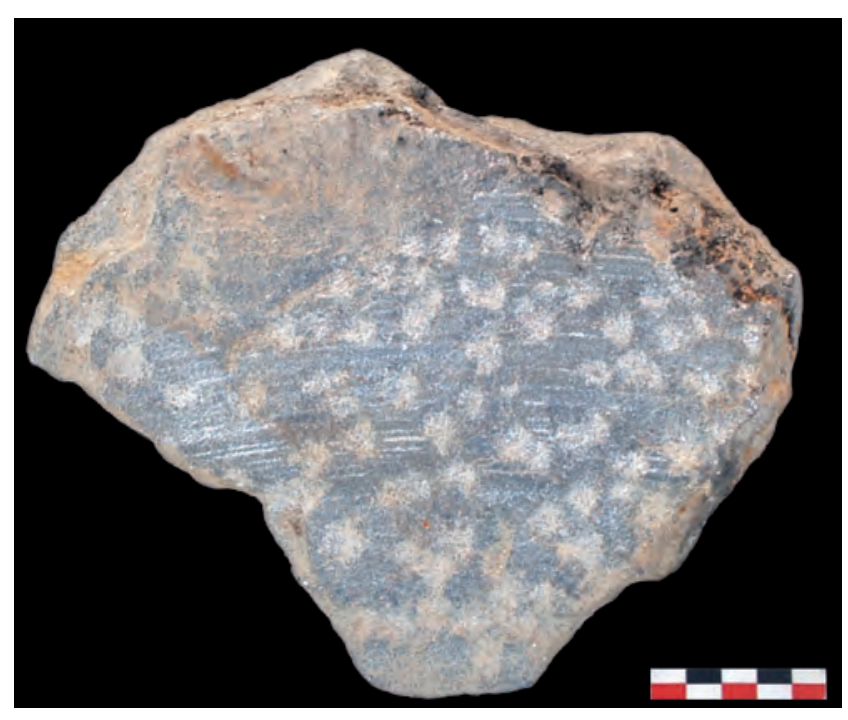

Fig. 16. Meule 2013-130. Piquetage.

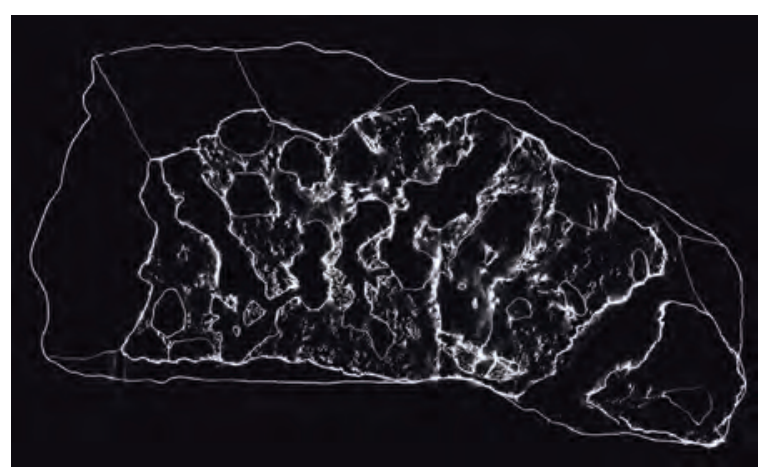

Fig. 18. Meule 2014-072 (vue du frottis). Piquetage.

\section{LE BROYAGE}

Après avoir décrit la meule à minerai «type» de ce corpus, tant avec sa forme que ses composants, il reste à la voir fonctionner. L'usure, le rhabillage et la fragmentation forment trois étapes concomitantes de l'évolution d'une meule. Enfin, nous achèverons ces réflexions en tentant d'estimer a minima le nombre de meules utilisées dans ce moulin.

\subsection{L'usure des meules}

Les meules sont utilisées de façon intensive, nous le remarquons avec l'étude des surfaces actives. La quasi-totalité des fragments du corpus 2031/2014 est striée; seuls 11 individus ne possèdent pas ces marques issues du passage du minerai. Les stries sont des sillons d'une épaisseur et d'une profondeur millimétrique variable issus du broyage de matériaux durs, plomb argentifère, gangue quartzo-barytique, et probablement quelques restes de roches encaissantes (gneiss, dolomie).

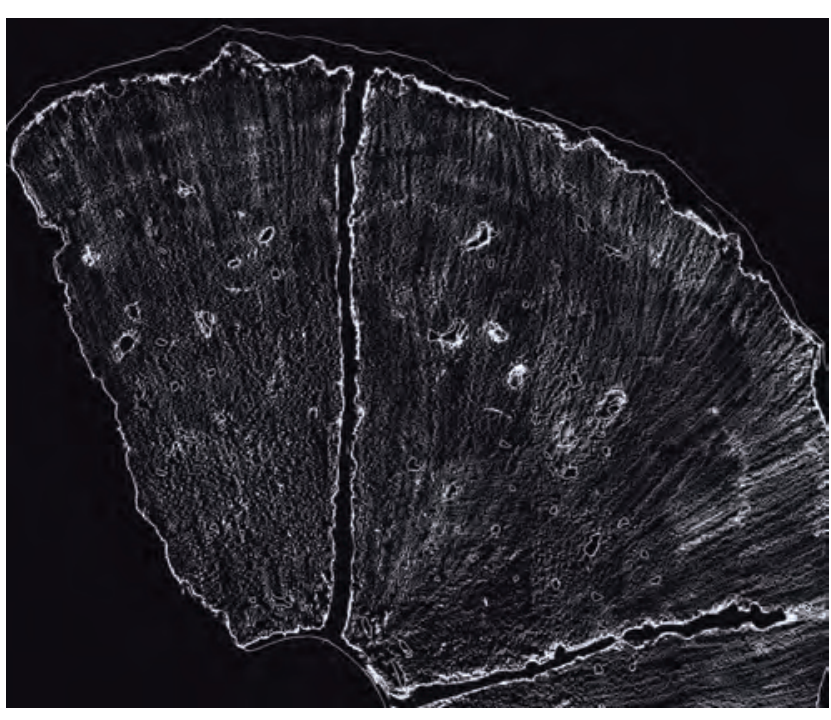

Fig. 17. Meule 2013-175 (vue du frottis). Piquetage et rayonnage continu.

Ces marques se répartissent sur les surfaces actives des meules en s'affinant, du cœur de la meule à sa feuillure. L'agencement des meules est à l'origine du processus : la forme des pierres, convexe pour la dormante et concave pour la tournante engendrent un écartement, la «lumière», plus important au niveau de l'œil, et cet écartement se réduit régulièrement jusqu'aux feuillures. Par conséquent, le minerai introduit dans l'œil a un module centimétrique, et ressort au niveau des feuillures dans un état millimétrique, sous forme de sable.

\subsection{Le rhabillage}

Le rhabillage est un procédé qui permet de redonner du mordant aux meules à chaque fois que les surfaces actives deviennent trop lisses, donc moins efficaces. Il consiste à inciser la surface active à coups perdus avec des marteaux spécifiques. Ce travail est généralement effectué par le meunier. Si le rhabillage des surfaces actives est très courant pour les meules à blé, nous en avons aussi quelques exemples pour le minerai.

En 2009, trois seulement avaient été repérés (2007-0-1510, 2009-1-020, 2009-1-052). Nous avions alors conclu à une pratique marginale. Parmi le corpus réuni en 2013/2014, douze fragments ont cette fois conservé des traces de piquetage sur leur surface active (2013-002/021/023/057/070/099/130/160 ; 2014$102 / 109 / 135 / 175 /)$. Il semble donc que la pratique du rhabillage des meules à coups perdus était bien effectuée sur les meules à minerai et qu'elle devait être fréquente. Elle concerne aussi bien les meules dormantes que les meules tournantes. Le faible nombre de fragments piquetés s'explique en partie par le fait que le rhabillage a pu être effacé par une dernière utilisation avant rejet. 


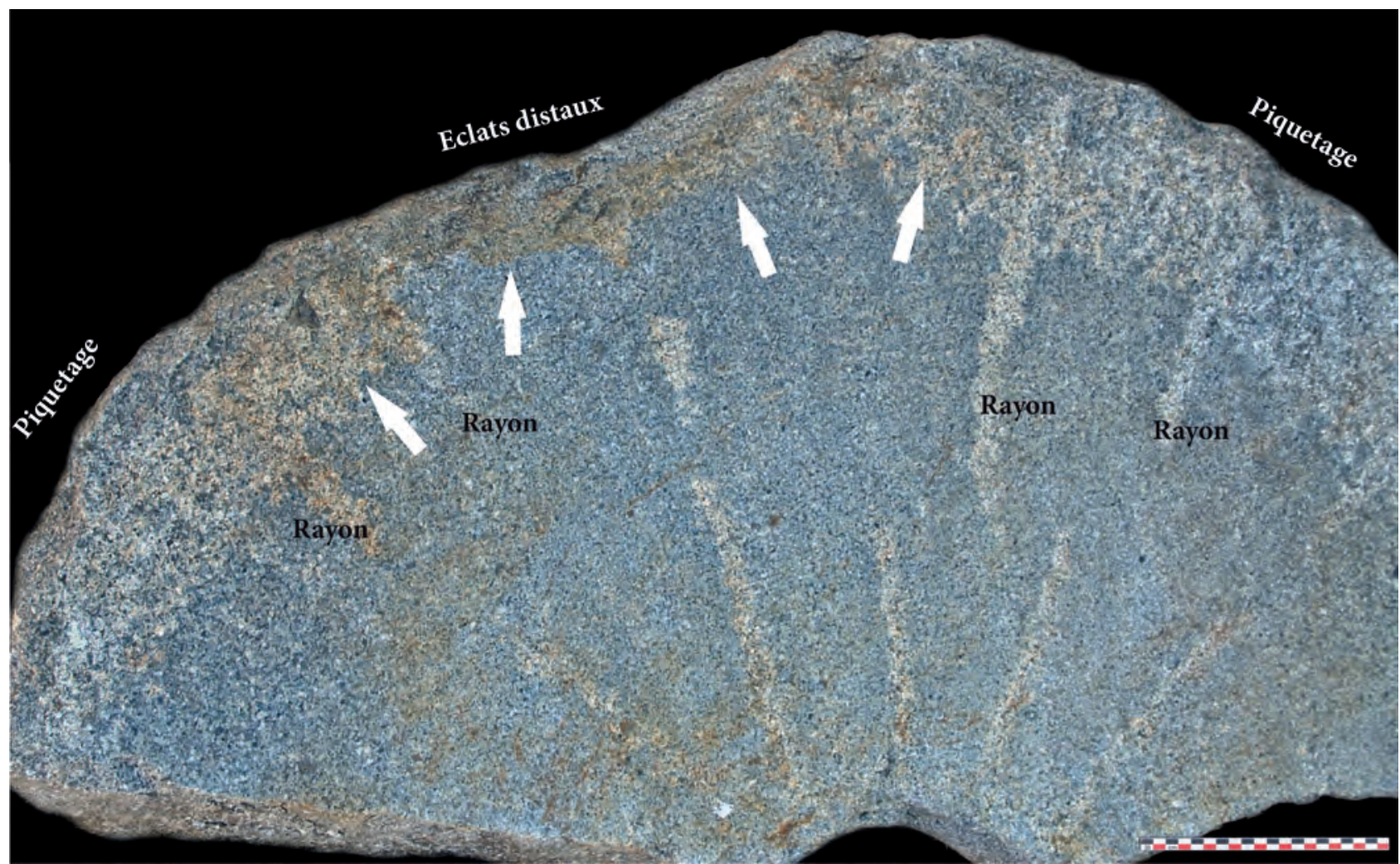

• Fig. 19. Meule 2013-001. Vue de la fragmentation distale.

- Fig. 20. Exemples d'éclats distaux.

Les meules mises au jour autour du moulin montrent que le piquetage était pratiqué sur l'ensemble de la surface active (2013-130/175) et sur ses extrémités (2013-070). Les fragments 2013-130 et 2013-175 sont de bon exemple du processus, on voit nettement que la surface active striée est recoupée par les coups de pics (fig. 16 et 17). Le rhabillage est fait dans un objectif identique au rayonnage. Les deux procédés sont utilisés de façon concomitante et l'un n'exclut pas l'autre. Dans le cas du fragment 2014-072 (fig. 18), on peut d'ailleurs s'interroger sur la frontière entre rayonnage et piquetage. Lorsque les coups de pics sont plus larges, on arrive à un résultat équivalent.

Rhabiller une meule nécessite de soulever la meule tournante à l'aide d'un système de levier. Aucune encoche latérale n'a été repérée sur les meules tournantes pour faciliter ce levage avec une potence pourvue d'une grande pince; on est dans la même situation que les meules du moulin de Thervay.

\subsection{La production d'éclats}

Au cours des campagnes 2013 et 2014, 309 éclats ont été rassemblés. En parallèle des stries de la surface active, le broyage marque les meules en fragmentant leur partie distale. L'extrémité de la feuillure est une zone fragile, car c'est un bord et que son épaisseur est plus fine. Le rayonnage conduit aussi à isoler aux extrémités des espaces qui

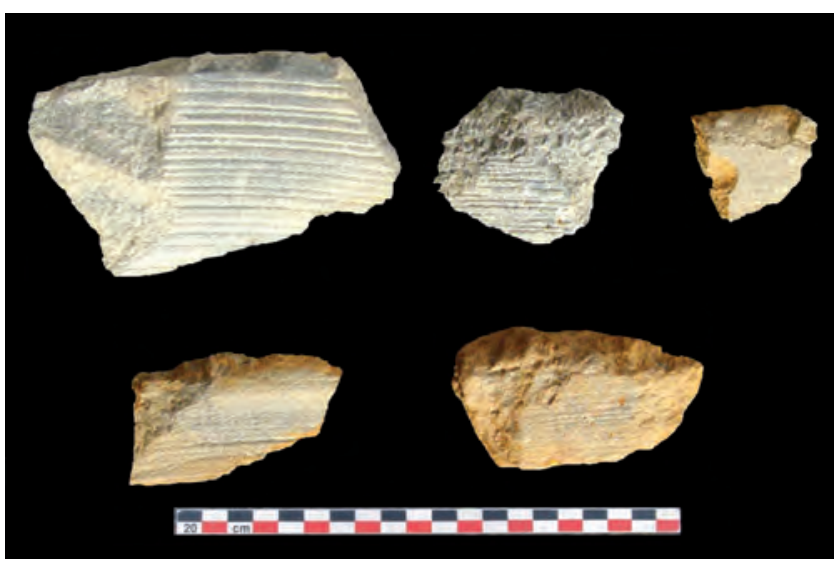

deviennent encore plus sensibles. La fragmentation s'effectue lorsque le moulin est en fonction. Les meules s'émiettent progressivement et projettent sur leur pourtour ces fragments de quelques centimètres de côté que l'on nomme éclat distal (fig. 19)

Les éclats distaux sont typologiquement tous identiques. Il s'agit de fragment de quelques centimètres de côté, généralement peu épais. Lorsqu'ils sont striés, ils le sont systématiquement avec des stries fines de type feuillure. Lorsqu'ils ne sont pas striés, ce qui est plutôt rare, leur surface active est polie. On reconnaît ces éclats par leurs stries, et par la roche, qui tranche généralement avec les autres matériaux (fig. 20). 


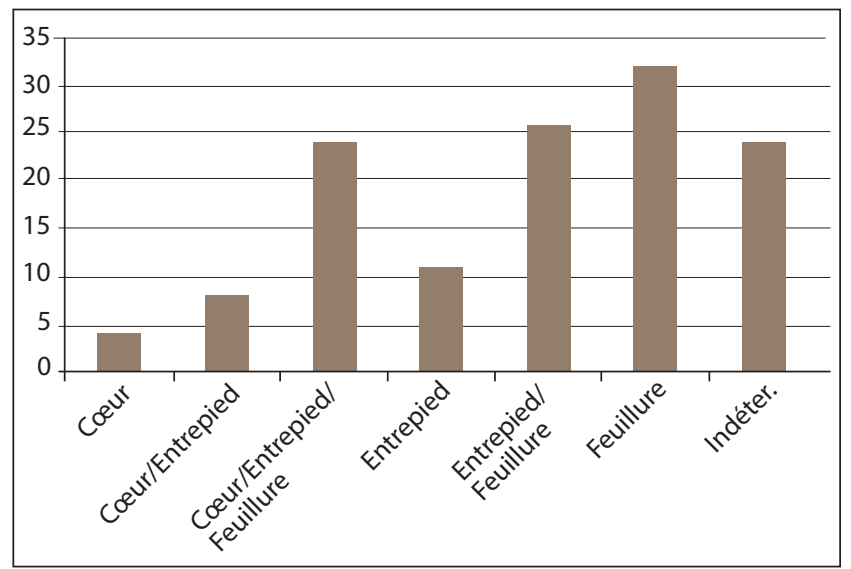

4 Fig. 21. Types de fragments conservés.

- Fig. 22. Modèle d'une surface active d'une meule à minerais striée.

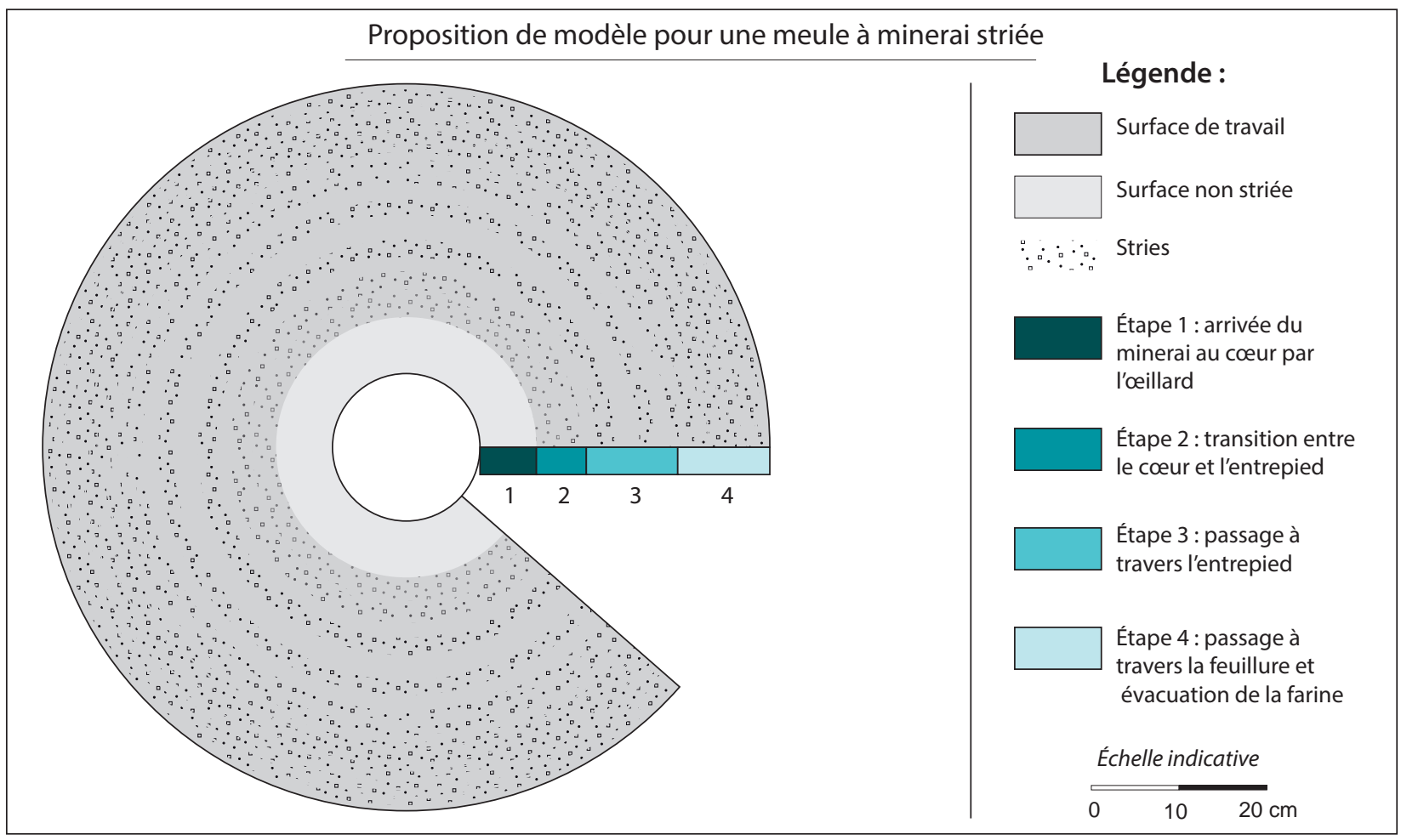

Actuellement, leur intérêt est double: ils permettent d'une part de mettre en évidence des espaces de broyage. Déposés au cours des rotations des meules, ils ne sont pas réemployés, et peu dispersés ensuite. En restant en position primaire, ils peuvent attester l'existence d'un broyage et approcher sa localisation, même en l'absence de structures conservées. D'autre part, ils permettent de suivre le trajet du minerai, du broyage au lavage. Comme nous en avons retrouvé plusieurs dans des structures de lavage, ces concentrations attestent que leur sable provient au moins partiellement d'un broyage, et non pas directement du concassage ${ }^{4}$.

4. Au Moyen Âge central la préparation du minerai passe par trois étapes principales : concassage (mortiers/percuteurs); broyage (moulin) et lavage

\subsection{Le problème du nombre de meules}

Déterminer un nombre minimum d'individus peut paraître une gageure lorsque l'on considère la fragmentation du corpus, d'autant plus si l'on intègre la possibilité d'avoir des meules assemblées. La première question qu'il convient de se poser, c'est si l'ensemble des fragments inventoriés est bien représentatif d'une meule à minerai, ou s'il y a eu des pertes qui empêcheraient toute estimation. Cela permet dans un second temps de calculer un nombre minimum théorique à partir notamment des surfaces actives.

(bassin de lavage). Le broyage et le lavage ne sont pas systématiques, ils permettent de traiter des modules centimétriques à millimétriques. 


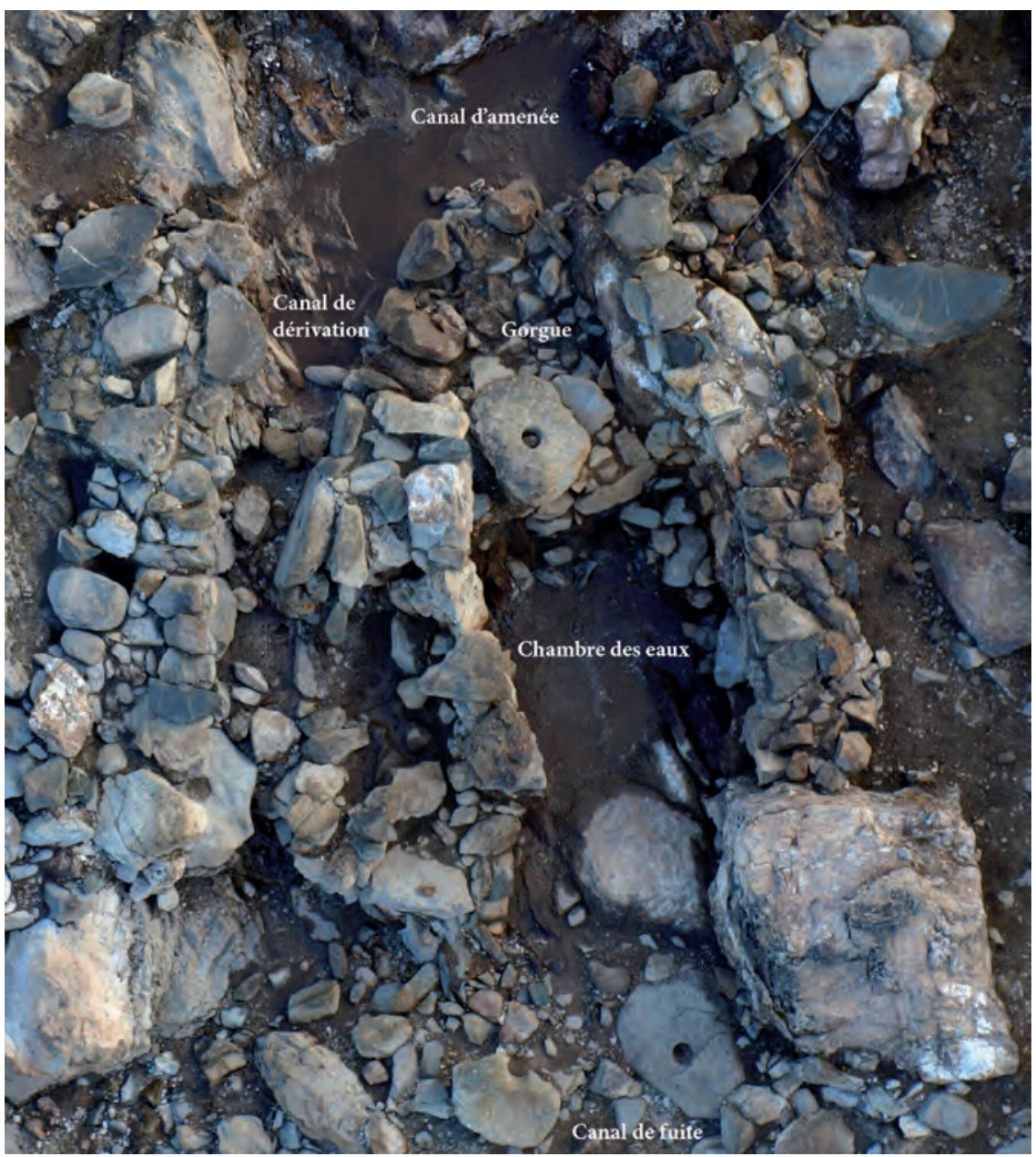

Fig. 23. Vue aérienne du moulin de Brandes (état 2013).

\subsubsection{Quelle représentativité?}

Lorsque les bords ou les œils sont partiellement conservés, on est en mesure de placer le fragment dans la meule, entre cœur, entrepied et feuillure. Cela étant, peu de fragments sont pourvus de ces indices. Pour les autres, il faut se pencher sur les stries. Ce sont leurs formes, leurs tailles qui vont nous permettre de classer la majeure partie des fragments (fig. 21). Le corpus 2013/2014 se compose d'une petite majorité de feuillures (trente-deux individus); suivent ensuite des fragments mixtes entrepied/feuillure (vingt-six individus) et les fragments tripartites (vingt-quatre individus). Les autres catégories sont moins représentées: les entrepieds seuls sont au nombre de onze, l'assemblage cœur/entrepied comprend huit individus, et les seuls cœurs, quatre. On observe ainsi une nette prédominance des fragments situés sur la feuillure de la meule. Si on cumule les données des premières catégories, on arrive pour la feuillure à 82 individus $(43,9 \%)$, pour l'entrepied à $69(36,9 \%)$, et pour le cœur à $36(19,3 \%)$.

Pour calculer les surfaces théoriques prises dans la meule par ces trois catégories, on s'est appuyé d'une part sur le modèle de meule striée, et d'autre part sur la moyenne des diamètres des meules et des œils. Ainsi une meule modèle aura un diamètre de $110 \mathrm{~cm}$, un œil modèle de $17 \mathrm{~cm}$ (fig. 22). La surface totale de cette meule est de $0,951 \mathrm{~m}^{2}$. Dans ce modèle, la feuillure a une surface de $0,445 \mathrm{~m}^{2}$, l'entrepied de 0,364 $\mathrm{m}^{2}$, le cœur de 0,119 $\mathrm{m}^{2}$ et l'œil de $0,023 \mathrm{~m}^{2}$. En réduisant ces données absolues en pourcentage, on aboutit à la séquence suivante : feuillure 46,8\%, entrepied $38,3 \%$, cœur $12,5 \%$, œil 2,4\%. La répartition des fragments retrouvés dans et autour du moulin correspond à ce modèle théorique. Les fragments rejetés et réunis seraient bien représentatifs d'une surface active complète.

\subsubsection{Le nombre minimum d'individus}

Si une meule, une demi-meule et un secteur comptent pour un individu (aucun ne recolle), on arrive à un NMI de vingt-deux meules. Pour évaluer les fragments divers, la méthode choisie a été d'additionner leurs surfaces actives. En confrontant ce nombre avec la surface active moyenne d'une meule $\left(0,951 \mathrm{~m}^{2}\right.$ avec œil, 0,928 $\mathrm{m}^{2}$ sans œil), on aboutit à un nombre minimum théorique. Sur 88 fragments 
N. MinVIELLE LAROUSSE

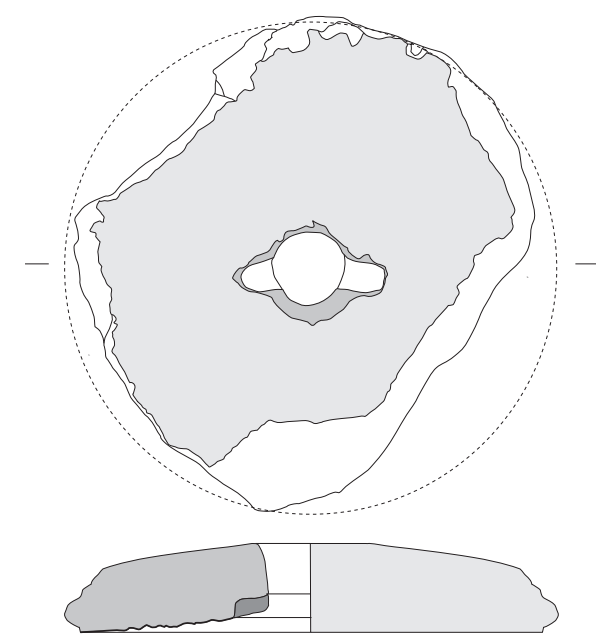

2013-196

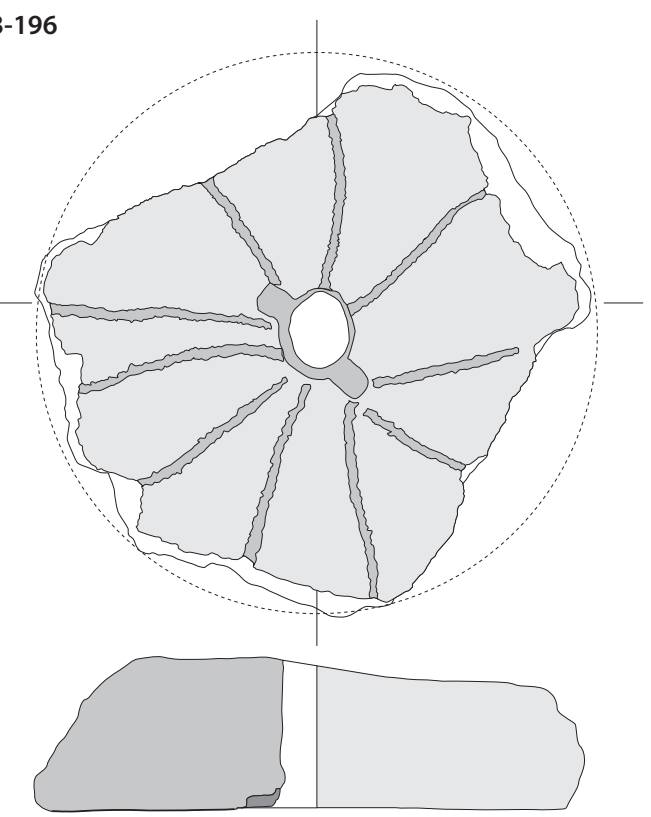

2014-174
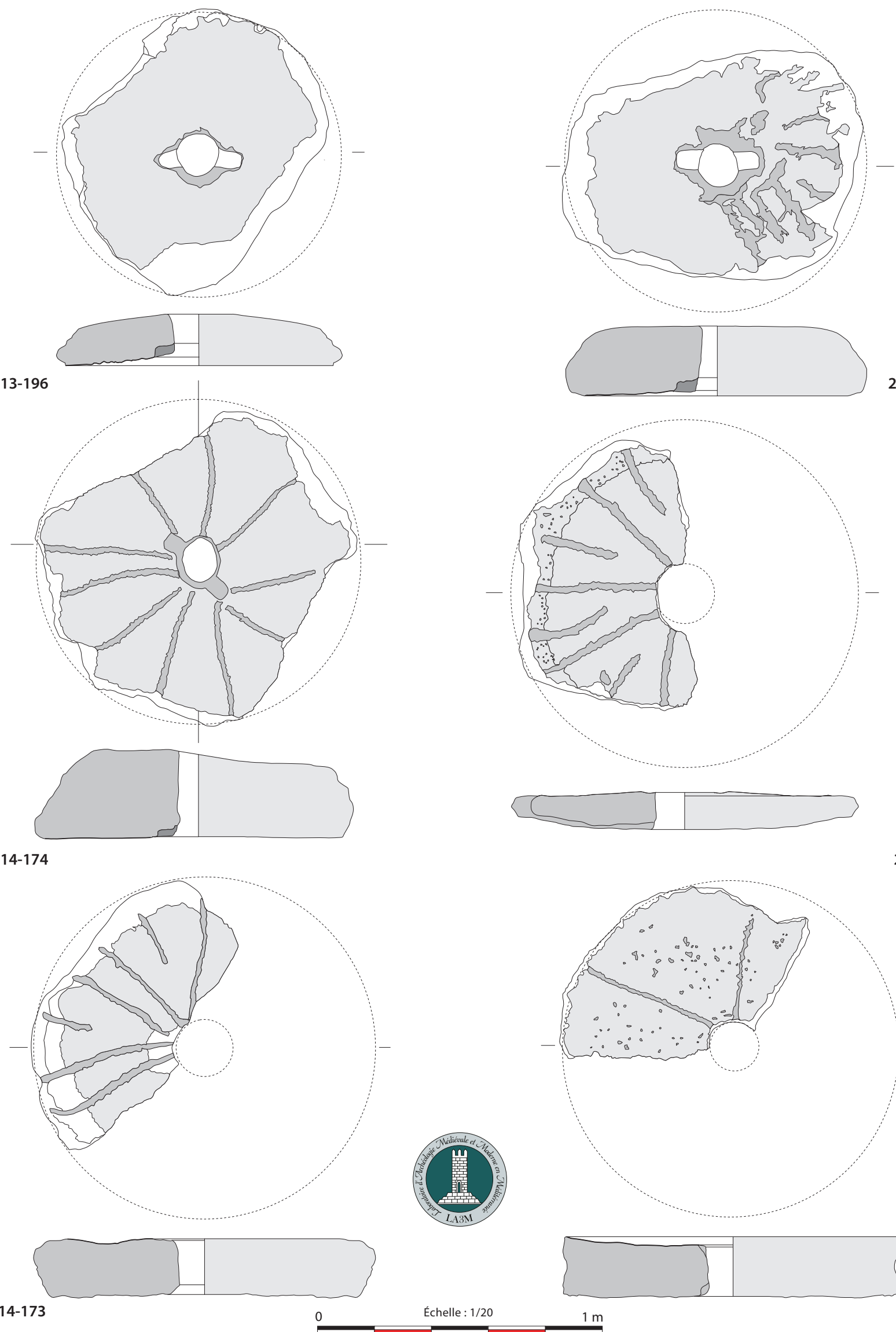

2014-163

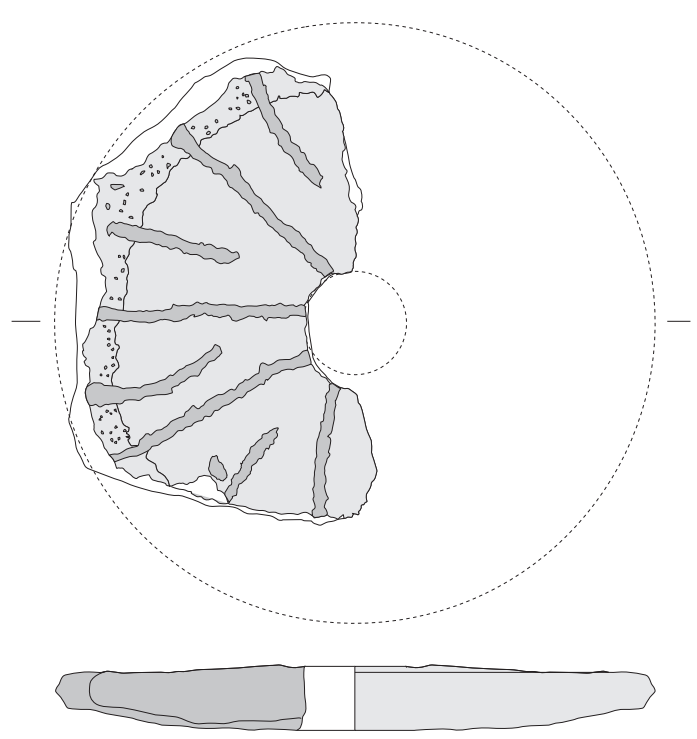

2013-070

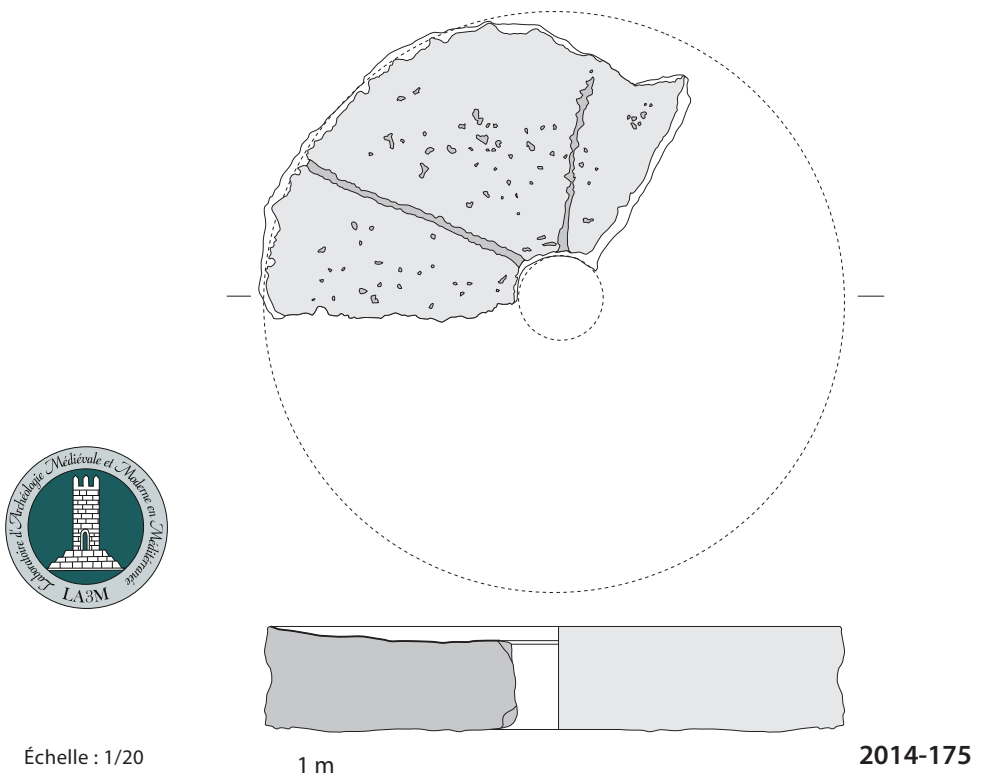

Fig. 24. Exemples de meules à minerais. 
LES MEULES DU MOULIN À MINERAI MÉDIÉVAL DE BRANDES-EN-OISANS
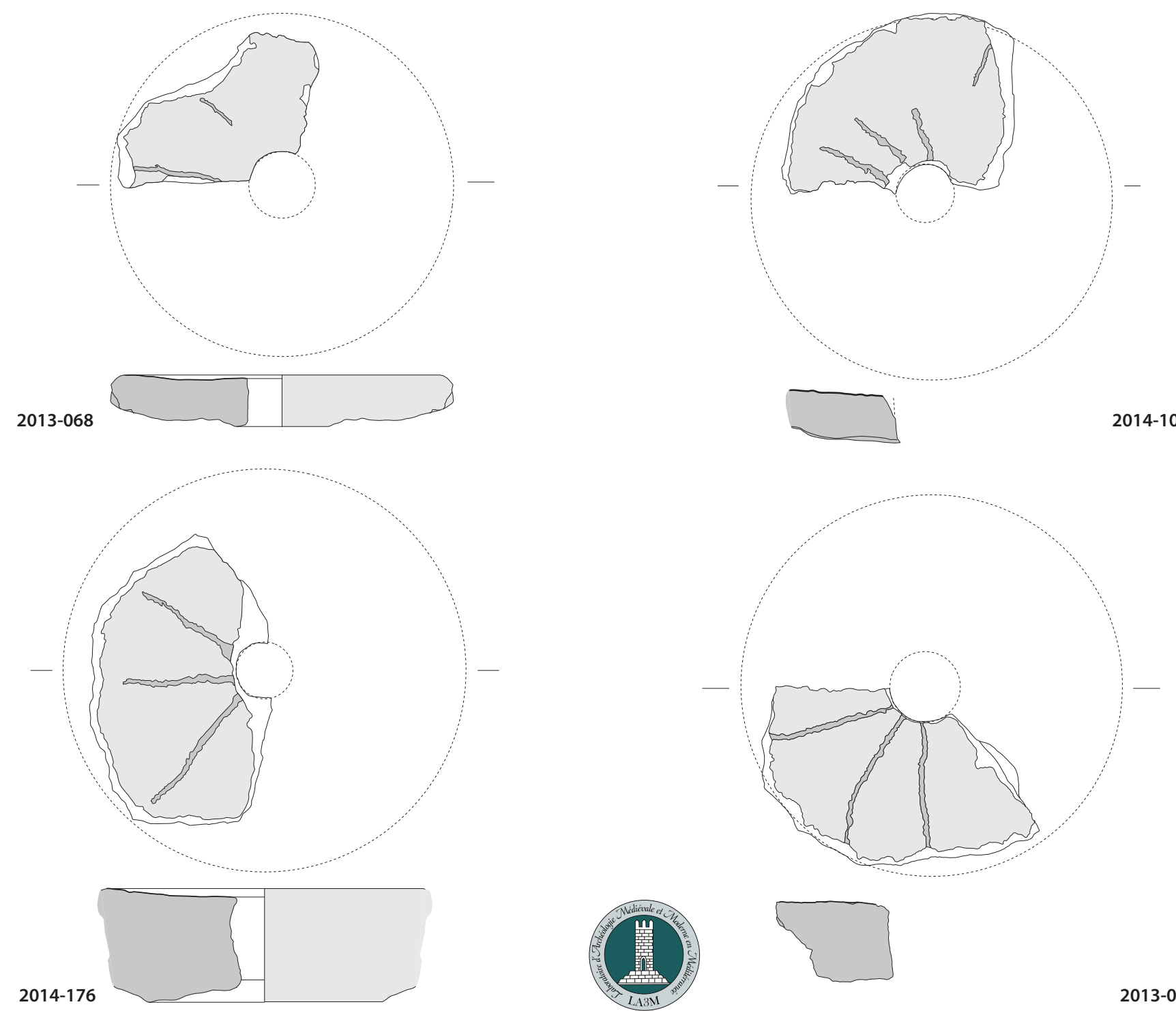

2014-103

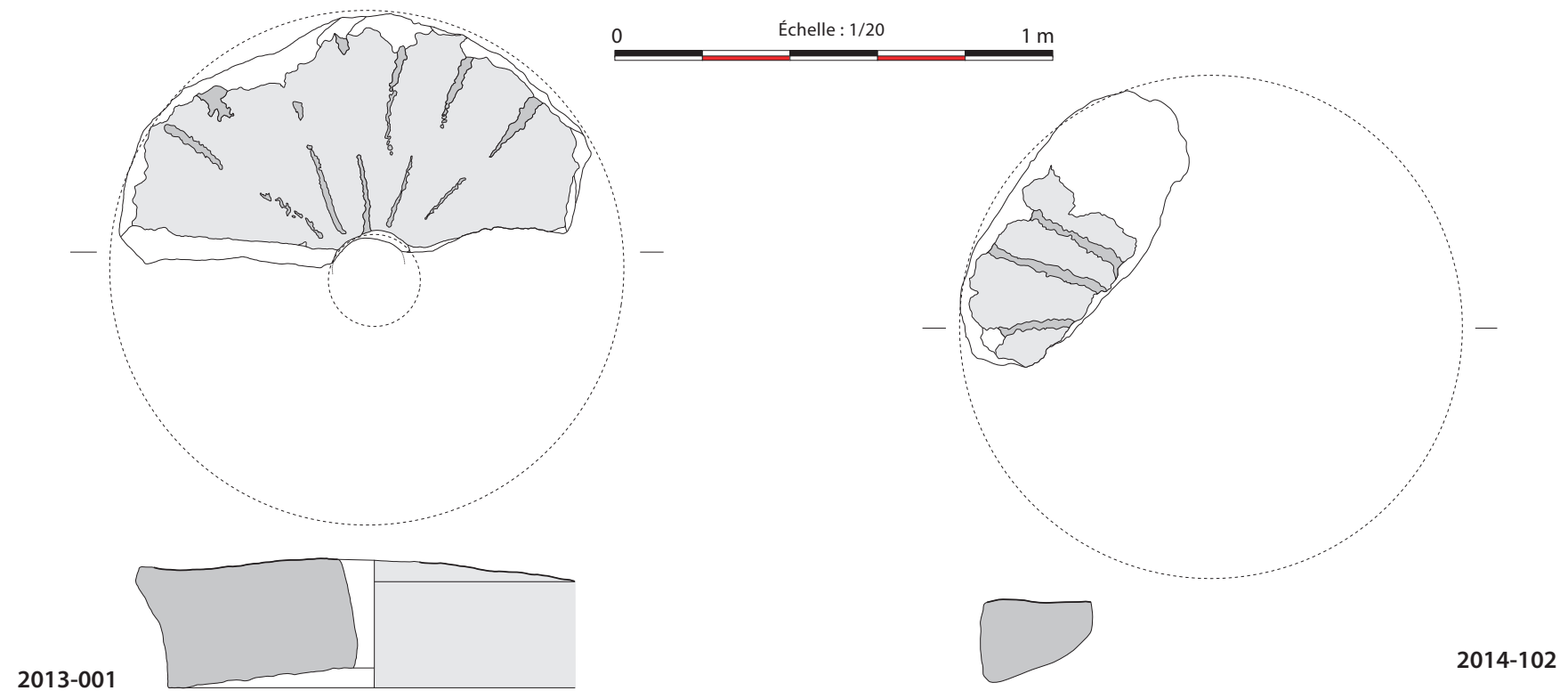

Fig. 25. Exemples de meules à minerais. 
N. MinVieLLE LAROUSSE
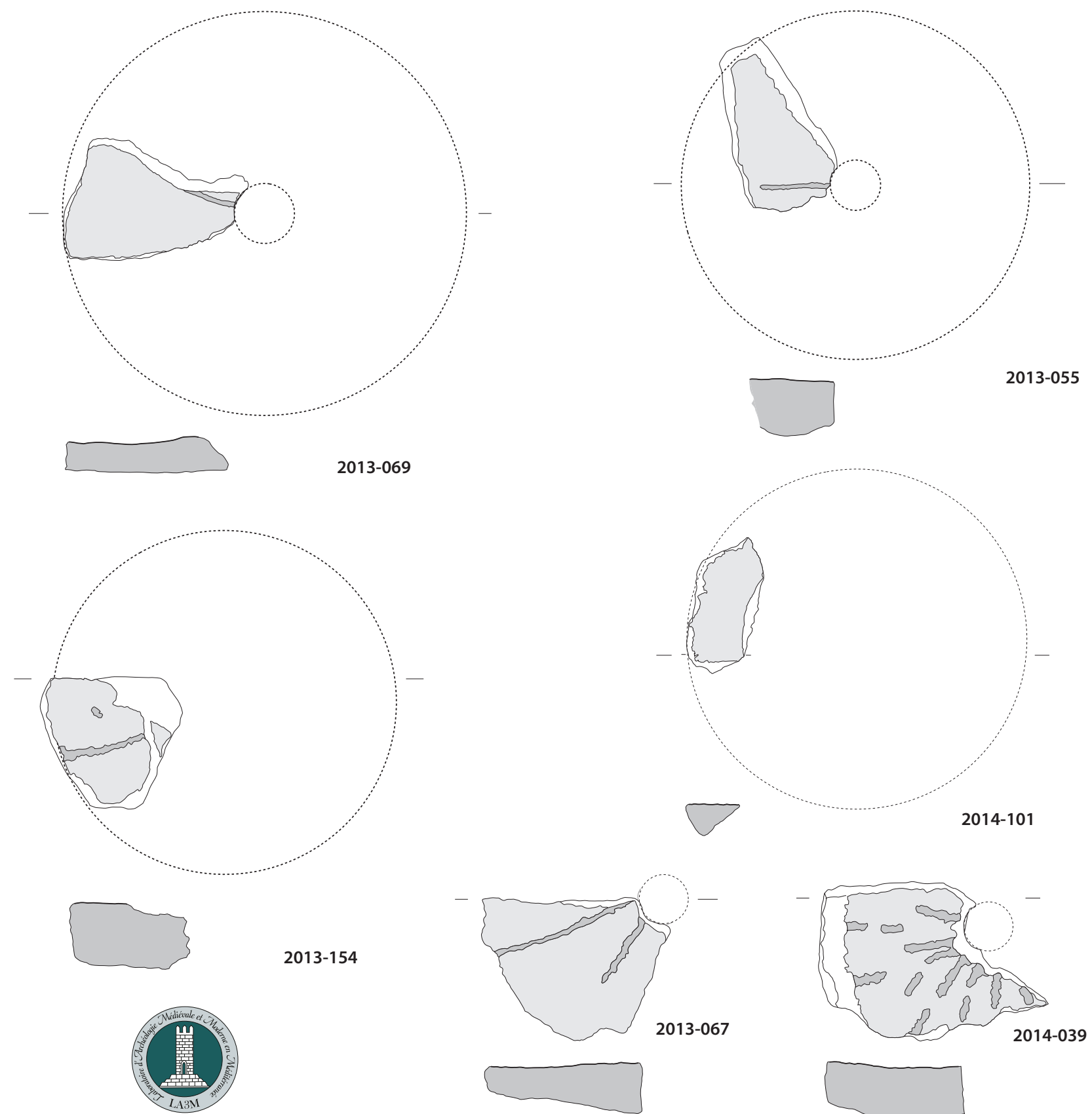

2013-154
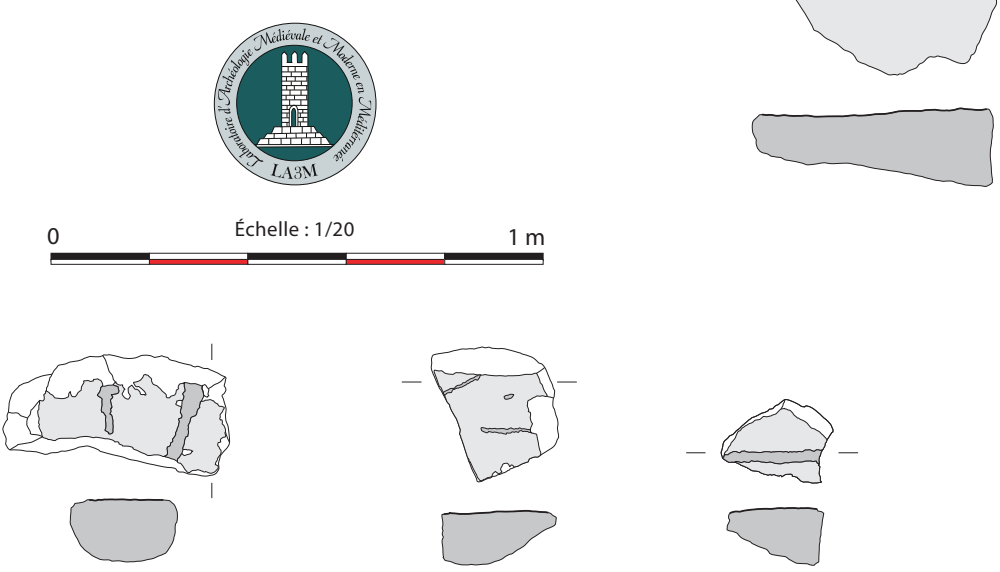

2014-039

Échelle : $1 / 20$

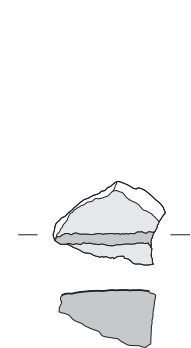

2014-111
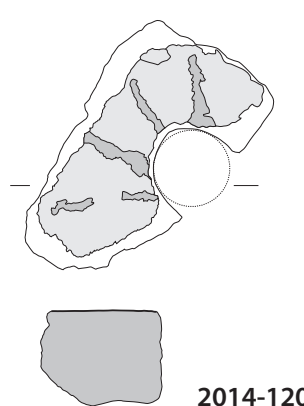

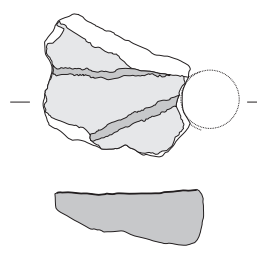

2014-121

Fig. 26. Exemples de meules à minerais. 
mesurés, la surface active totale est de 2,605 $\mathrm{m}^{2}$ : l'ensemble de ces fragments équivaut donc au minimum à trois meules $(2,8)$. Le nombre minimum d'individus, toutes US confondues, serait ainsi de vingt-cinq meules.

Cette estimation est partie du présupposé que les fragments pussent recoller, ou être intégrés dans des meules assemblées. C'est probablement loin d'être systématique. Nous n'avons pas pu travailler sur des recollages, la masse de certaines pièces était contraignante, et la fragmentation des autres les rendait hasardeux et chronophage. Le NMI présenté est donc une estimation très basse.

\section{CONCLUSION}

Qu'est-ce donc qu'une meule à minerai au XIII ${ }^{\mathrm{e}} \mathrm{s}$. ? Elle est déjà, d'après ce que l'on connaît, exclusivement hydraulique (fig. 24, 25 et 26). Son diamètre va de 60 à $130 \mathrm{~cm}$, plutôt $100 \mathrm{~cm}$ en moyenne pour une épaisseur avant utilisation d'une trentaine de centimètres. Les matériaux utilisés sont locaux, on privilégie un approvisionnement immédiat, si possible sur blocs erratiques. L'œil, circulaire et large de 15 à $20 \mathrm{~cm}$, est traversant. La meule tournante se différencie de la meule dormante par l'encoche de l'anille, grossièrement quadrangulaire, d'une trentaine de centimètres de longueur et de $5 \mathrm{~cm}$ environ de largeur; ou par la courbure de leur surface active, convexe pour la dormante, concave pour la tournante. Contrairement aux pierres à pain contemporaines, les meules à minerai sont majoritairement rayonnées, que ce soit de façon continue ou discontinue, parfois aléatoirement. Peu espacées en raison d'une faible conicité, les surfaces actives sont toutes ou presque striées. La réduction du module du minerai se suit avec leur épaisseur, d'abord par le cœur, inexistante ou grossière ; puis l'entrepied, centimétrique; enfin la feuillure, millimétrique. L'usure est de fait importante: les meules fondent et s'émiettent progressivement alors que les rhabillages à coups perdus se succèdent pour leur redonner du mordant et prolonger leur activité. Lorsqu'une meule devient obsolète sur une face, il n'est pas rare de la retourner sur l'autre pour la finir. Il est également possible que des fragments ou carreaux soient assemblés en complément des meules monolithes, soit dès l'origine, soit une fois de plus pour recycler les rebuts.
Nous les avons trouvées usées et fragmentées, ce qui accentue sans doute leur aspect dissymétrique et augmente les cas de recyclage. Néanmoins, leur aspect correspond finalement bien à celui du moulin. Construit rapidement, avec des matériaux de récupération ou immédiatement disponibles, il a été placé de façon opportuniste sur une faille gneiss/dolomie bien pratique pour mettre en place le système hydraulique. S'il nous manque la plupart des structures de bois pour en avoir une vue complète, il apparaît comme une construction utilitaire sans aucune fioriture. La répartition des fragments et éclats de meules a contribué à interpréter ses structures, tout comme ces dernières expliquent peut-être la dissymétrie récurrente des meules (fig. 23).

Ce portrait n'est pas une fin en soi, il a pour objectif à moyen terme de contribuer à deux thèmes de l'histoire des techniques. Sur la chaîne opératoire des métaux polymétalliques, tout le processus d'enrichissement du minerai a maintenant un référentiel archéologique. Dernier fouillé, le broyage fait depuis peu l'objet d'approches expérimentales sur la plate-forme de Melle (TÉREYGEOL dir., 2012) et sera intégré aux synthèses prévues sur le traitement du minerai à Brandes-en-Oisans. Sur les problématiques relatives aux moulins et aux meules en général, il n'y a encore que peu d'exemples d'usages industriels (BENOÎT, 1998; BUCHSENSCHUTZ et alii, 2011; BENOÎT, 2014). Le cas du minerai s'intègre dans ces recherches et nous questionne en particulier sur le passage d'un broyage qui serait manuel durant l'Antiquité à un broyage hydraulique au Moyen Âge. Est-ce une innovation ou encore un état de la recherche?

\section{Remerciements}

Il est agréable de remercier ici tous ceux qui nous ont apporté leur aide dans cette étude: Henri Amouric, Marie-Christine Bailly-Maître, Béatrice Cauuet, Luc Jaccottey, Flavien Perazza, Florian Téreygéol ; et notamment Stéphane Rouméjon pour la détermination des roches ainsi que les fouilleurs du Groupe d'Études des Mines Anciennes (GEMA) qui ont été chargés, sous la conduite de Luc Jaccottey, de l'enregistrement des meules sur le terrain: Natacha Barré, Niels Fourchet, Mélodie Larue, Aude Marguet. 